\title{
SOLAR AND STELLAR SEISMOLOGY
}

\author{
KEN G. LIB B RECHT \\ Solar Astronomy 264-33, California Institute of Technology, Pasadena, CA 91125, U.S.A.
}

(Received 15 October, 1987)

\begin{abstract}
This article reviews solar and stellar seismology, with emphasis on the enormous progress which has been made recently in the observation and understanding of solar p-modes. Precision measurements of $p$-mode frequencies and frequency splittings allow a greater understanding of the structure of the solar interior, while p-mode amplitudes and linewidths shed light on the mode excitation mechanism, which is probably stochastic excitation by turbulent convection. The prospects for making similar measurements on other stars are also discussed.
\end{abstract}

\section{Introduction and History}

This review is primarily about the observed oscillations of the Sun, the physics underlying these oscillations, and their impact on our understanding of the Sun, particularly the solar interior. Past reviews include Deubner and Gough (1984), ChristensenDalsgaard et al. (1985), Leibacher et al. (1985), Brown et al. (1986), and Toomre (1986). Present and future observations of similar oscillations on other stars are also discussed.

Oscillations of the solar surface were first observed by Leighton et al. (1962) and by Evans and Michard (1962). These authors found a 5-min correlation of solar Doppler velocities, and further observations showed that all points on the Sun's surface exhibited vertical oscillations with periods of roughly $5 \mathrm{~min}$ coherence times of order $20 \mathrm{~min}$, and amplitudes of order $1 \mathrm{~km} \mathrm{~s}^{-1}$. For many years the oscillations were believed to be an incoherent surface phenomenon, until it was suggested by Ulrich (1970) and independently by Leibacher and Stein (1971) that the oscillations were the surface manifestation of acoustic modes which were trapped in the solar interior. Ulrich, and subsequently Ando and Osaki (1975), calculated a dispersion relation for the trapped modes, and predicted that the surface oscillation power would be concentrated in ridges when plotted as a function of horizontal wave number and temporal frequency, in a so-called $k-\omega$ power diagram. These predictions were soon confirmed by Deubner (1975), and a modern version of such a power plot, here an $l-v$ diagram, with $l \simeq k R_{\odot}$ and $v=\omega / 2 \pi$, is shown in Figure 1.

With this new theoretical understanding of the oscillation phenomenon, backed up by the observations, came the realization that what had appeared in the 1960s to be incoherent surface oscillations was in reality the incoherent superposition of millions of uncoupled, long-lived, global solar oscillation modes, the Sun's normal modes of oscillation. 


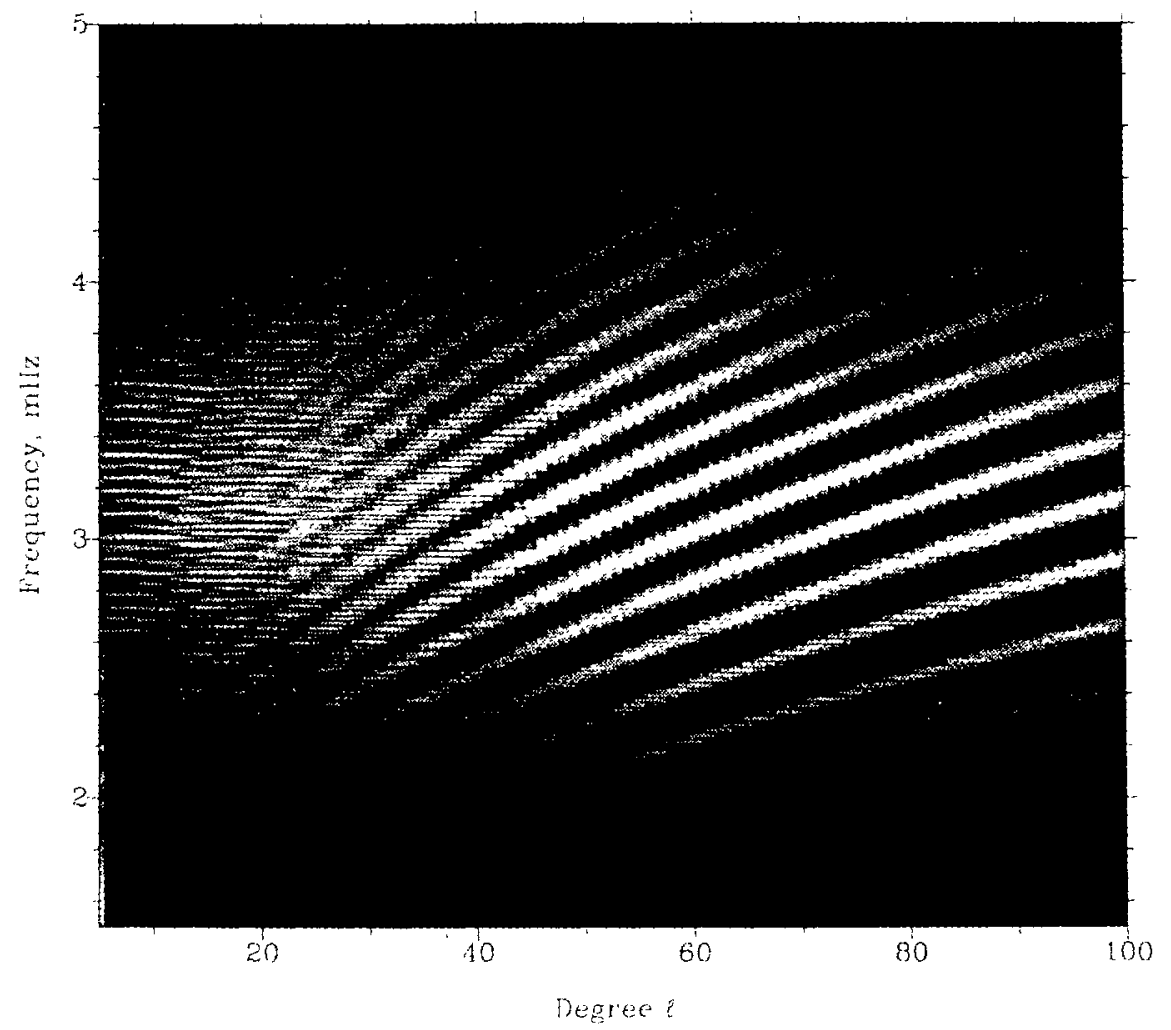

Fig. 1. Plot of the power in the solar oscillations as a function of spherical-harmonic degree $l$ and temporal frequency $v$, obtained using 12 days of data from Big Bear Solar Observatory in California. Each ridge in this diagram contains oscillations with a fixed number of radial nodes $n$. (From Duvall et al., 1988.)

Observations of the Doppler shift of integrated sunlight (i.e., without imaging) by Claverie et al. (1979) were also found to exhibit five-minute oscillations, and individual normal modes were first isolated by Grec et al. $(1980,1983)$ using a five-day time-series of measurements of the Doppler shift of integrated sunlight taken from the South Pole. Only the lowest $l$-values are not averaged to zero in these measurements, and the very sharp peaks in the power spectrum of the time-series, show in Figure 2, proved that the oscillations were indeed long-lived global normal modes.

\section{Theory}

Because the observed solar oscillations consist of small amplitude, high $Q$ oscillation modes, they are well described by a theory of linear adiabatic oscillations of a spherical Sun. The perturbation of a scalar quantity, for example pressure, resulting from the excitation of a single oscillation mode, can be characterized by three 'quantum' numbers $n, l$, and $m$ :

$$
\delta p_{n l m}=\operatorname{Re}\left[\delta p_{n l}(r) Y_{l}^{m}(\theta, \phi) e^{i \omega_{n l m} t}\right],
$$

where $\delta p_{n l}(r)$ is a radial wave function with $n$ radial nodes, $Y_{l}^{m}(\theta, \phi)$ is a spherical 


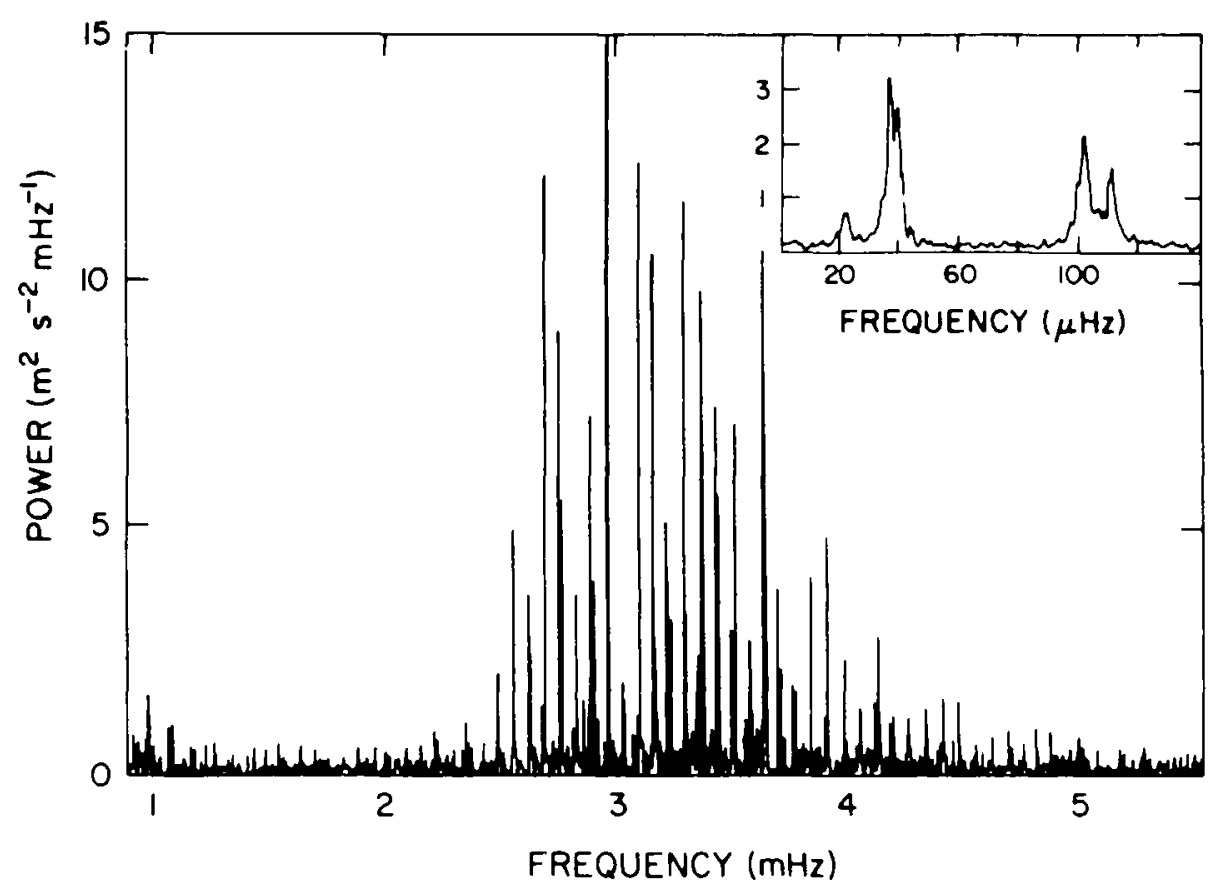

Fig. 2. An observed power spectrum of low $l$ p-modes, resulting from a 5-day continuous timestring of integrated sunlight radial velocity measurements, obtained at the geographic South Pole by Grec et al. (1983). Most of the power is concentrated in a few dozen very narrow peaks, where each peak corresponds to an oscillation mode with a particular value of radial order $n$ and spherical harmonic degree $l$. The largest amplitudes seen are about $20 \mathrm{~cm} \mathrm{~s}^{-1}$. The inset figure is a superposed frequency diagram, in which the spectrum has been folded on itself with a repetition period of $136 \mu \mathrm{Hz}$, demonstrating the regular spacing of features within the spectrum predicted by asymptotic theory. The four resulting peaks (from left to right) correspond to oscillations with $l$ of $3,1,2$, and 0. (From Brown et al., 1986.)

harmonic with degree $l$ and azimuthal order $m$, and $\omega_{n l m}$ is the angular frequency of the mode. For a perfectly spherical Sun, the mode frequency cannot depend on $m$, but as we see below this degeneracy is lifted by solar rotation. The fluid displacement is similarly given by

$$
\begin{aligned}
\xi_{n l m}= & \operatorname{Re}\left\{\left[R_{n l}(r) Y_{l}^{m}(\theta, \phi) \hat{r}+\Theta_{n l}(r)\left(\frac{\partial Y_{l}^{m}}{\partial \theta} \hat{\theta}+\frac{1}{\sin \theta} \frac{\partial Y_{l}^{m}}{\partial \phi} \hat{\phi}\right)\right] \times\right. \\
& \left.\times \exp \left(-i \omega_{n l m} t\right)\right\} .
\end{aligned}
$$

The linearized fluid equations, including the momentum and energy equations, the equation of continuity, and the equation of state, form a fourth-order system of ordinary differential equations for the eigenvalues $\omega_{n l m}$, which is normally solved numerically using an existing solar model. However, to get a physical feeling for the solutions of these equations, we follow the example originally set by Lamb (1932) for adiabatic oscillations of a stratified atmosphere, recently portrayed by Deubner and Gough (1984) for the solar case. Neglecting the local effects of the spherical geometry, and using the Cowling 
approximation, the equations of motion can be approximately written as

$$
\Phi^{\prime \prime}+K^{2} \Phi=0
$$

where $\Phi=\rho^{1 / 2} c^{2} \operatorname{div} \xi, \rho$, and $c$ are the density and sound speed of the equilibrium state, and a prime denotes differentiation with respect to $r$. The vertical component of the local wave number $K$ is given by

$$
K^{2}=\frac{\omega^{2}-\omega_{c}^{2}}{c^{2}}+\frac{l(l+1)}{r^{2}}\left(\frac{N^{2}}{\omega^{2}}-1\right)
$$

where

$$
\omega_{c}^{2}=\frac{c^{2}}{4 H^{2}}\left(1-2 H^{\prime}\right)
$$

is the acoustic cutoff frequency, $H$ is the density scale height,

$$
N^{2}=g\left(\frac{1}{H}-\frac{g}{c^{2}}\right)
$$

is the square of the buoyancy (Brunt-Väisälä) frequency, and $g$ is the acceleration due to gravity.

We can rewrite the above in the form

$$
c^{2} K^{2}=\omega^{2}\left(1-\frac{\omega_{+}^{2}}{\omega^{2}}\right)\left(1-\frac{\omega_{-}^{2}}{\omega^{2}}\right)
$$

where

$$
\omega_{ \pm}^{2}=\frac{1}{2}\left(S_{l}^{2}+\omega_{c}^{2}\right) \pm\left[\frac{1}{4}\left(S_{l}^{2}+\omega_{c}^{2}\right)^{2}-N^{2} S_{l}^{2}\right]^{1 / 2}
$$

with

$$
S_{l}=[l(l+1)]^{1 / 2} \frac{c}{r} .
$$

If solutions to the above are to be wavelike, we must have $K^{2}>0$, which occurs either when $\omega_{ \pm}$are real and (a) $\omega^{2}>\omega_{-}^{2}$ and $\omega^{2}>\omega_{+}^{2}$ or (b) $\omega^{2}<\omega_{-}^{2}$ and $\omega^{2}<\omega_{+}^{2}$. The two cases (a) and (b) describe two classes of normal modes of the Sun: the first is the acoustic or p-modes, for which the restoring force is predominantly pressure, and the second is the gravity or g-modes, for which buoyancy is the principle restoring force.

The critical frequencies $\omega_{+}$and $\omega_{-}$are plotted in Figure 3 . This propagation diagram shows graphically how waves can be trapped inside the solar interior to form standing waves, or equivalently normal modes. The p-modes, for example, can propagate inside the Sun, but are reflected from an outer boundary at the solar surface and from an inner boundary which depends on frequency and $l$. The g-modes can propagate in the deep solar interior, but are reflected at an outer boundary at the base of the solar convention zone. The so-called chromospheric modes trapped above $h=0$ (see Figure 3) have not 


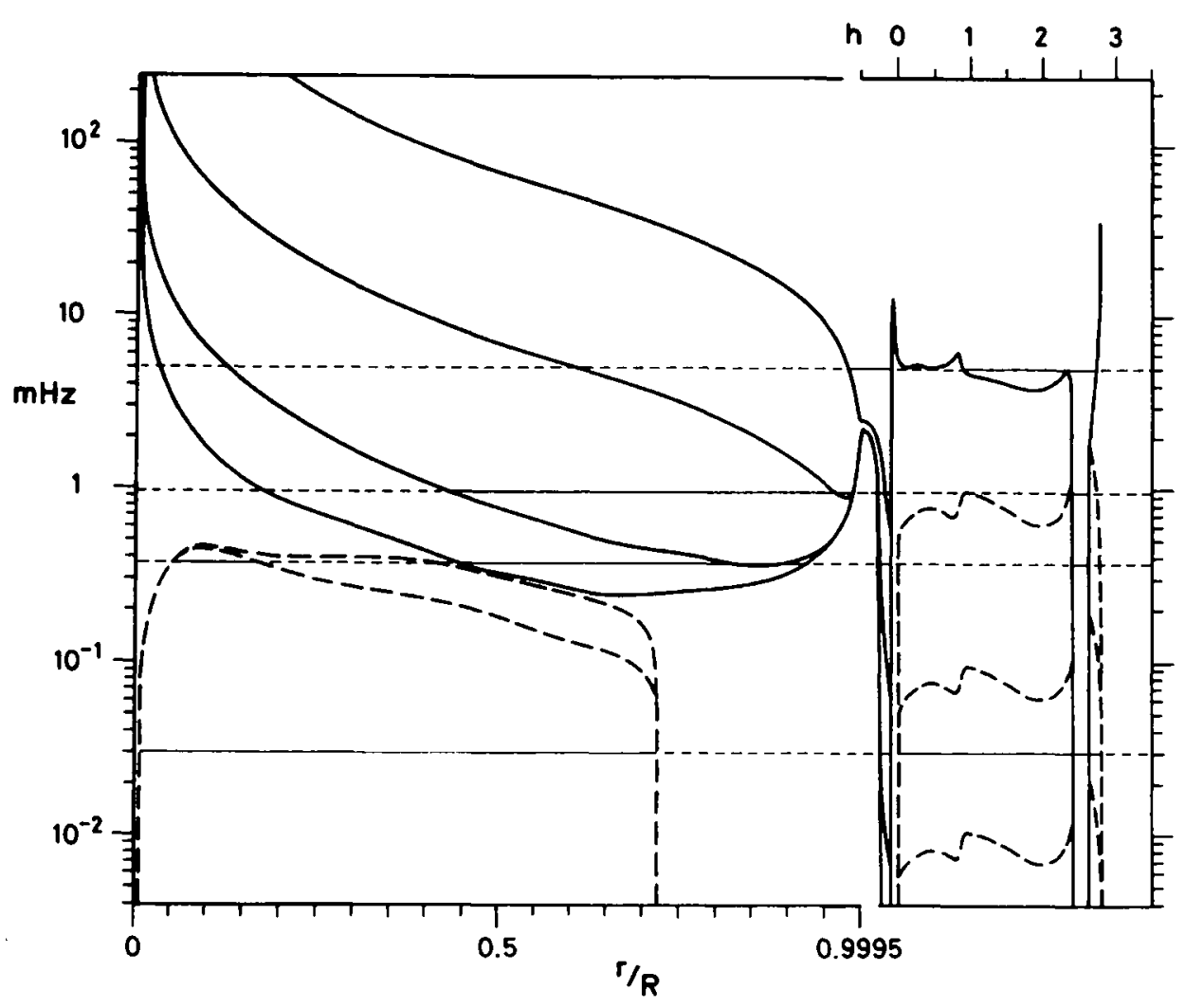

Fig. 3. Propagation diagram for a standard solar model. Solid curves represent $\omega_{+} / 2 \pi$, and dashed curves $\omega_{-} / 2 \pi$, in the regions where the critical frequencies $\omega_{ \pm}$are real. Propagation at any frequency is possible where $\omega_{ \pm}$are complex. The lower abscissa scale extends to $r / R_{\odot}=0.9995$; beyond that value, the scale is expanded by a factor of 100 , and is indicated on the upper boundary of the diagram, where $h$ is the height above the photosphere measured in units of $10^{-3} R_{\odot}$. The curves $\omega_{ \pm}$are for $l=1,5,50$ and 500. In all cases, $\omega_{ \pm}$are increasing functions of $l$ at fixed $r / R_{\odot}$, which permits the identification of the curves. In the interior the $\omega_{-}$curves for $l=5,50$, and 500 are essentially indistinguishable, as are all four $\omega_{+}$curves in the atmosphere, where $\omega_{+} \simeq \omega_{c}$. The thin horizontal lines represent trapped normal modes; they are continuous in zones of propagation and dashed in evanescent regions. (From Deubner and Gough,

1984.)

been unambiguously observed, and it is unlikely that these solutions represent global oscillation modes, owing to inhomogeneities in the chromosphere. Therefore, we will confine our discussion here to modes trapped beneath the photosphere, where the condition for a normal mode is essentially that an integral number of half wavelengths fit between the inner and outer turning points, or

$$
\int_{r_{\text {in }}}^{r_{\text {out }}} K \mathrm{~d} r \simeq n \pi .
$$

The p-modes are reflected at or below the photosphere if their frequency is below the acoustic cutoff frequency, roughly $v_{c} \simeq 5.5 \mathrm{mHz}$. Above this frequency the modes are not trapped inside the Sun and, therefore, quickly dissipate their energy in the chromosphere. Note that the turning point for high-frequency modes is higher in the 
atmosphere than for low-frequency modes, and that modes like the lowest frequency p-mode ( $l=0, n=1$, with a period of order $60 \mathrm{~min}$ ) are trapped far below the photosphere and, therefore, have not yet been observed. The inner turning point depends both on $l$ and frequency, and is roughly given by $r_{\text {in }} \simeq c\left(r_{\text {in }}\right) l / \omega$. Thus low $l$ p-modes penetrate more deeply into the solar interior than high $l$ modes of the same frequency, while the outer boundary condition does not vary appreciably with $l$, a result which is shown schematically by the ray diagram in Figure 4 . This $l$ dependence can be exploited to determine the radial dependence of some interior properties of the Sun, including the sound speed and the rotation rate, as we describe below. Note also from Figure 4 that the p-mode displacements are predominantly radial at the solar surface, owing to upward refraction since the sound speed increases with depth.

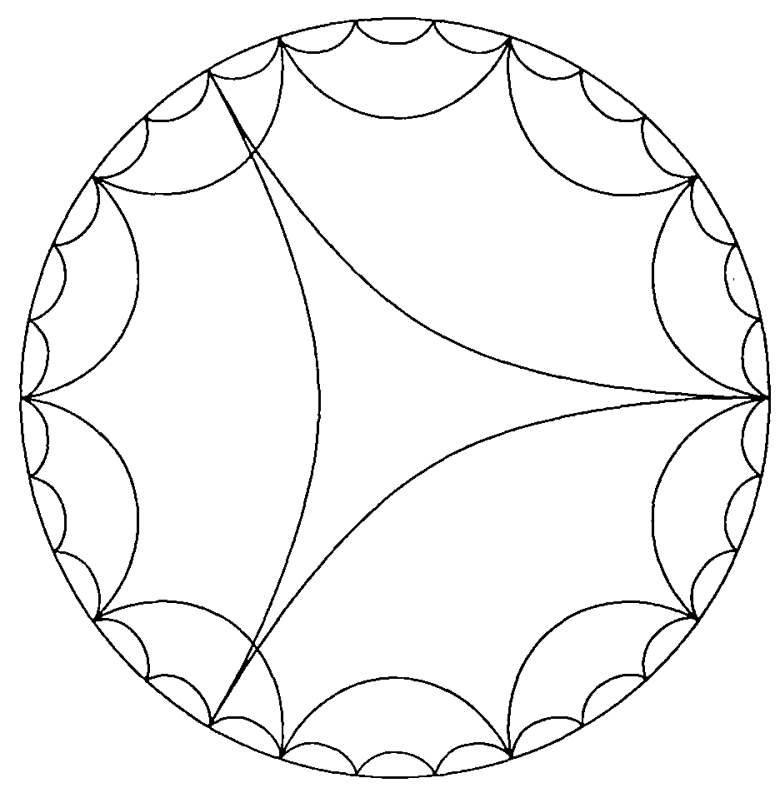

Fig. 4. Ray approximation for acoustic waves trapped in the solar interior. The circle represents the solar surface, and standing waves with $l=3,10$, and 30 are plotted. This diagram shows schematically that lower $l$ modes penetrate more deeply into the solar interior, and that the wave vector (and so the fluid displacement) is predominantly vertical at the solar surface. (Adapted from Toomre, 1986.)

An asymptotic expression for the mode frequencies when $n / l \gg 1$ has been derived by Tassoul (1980), which gives

$$
v_{n l} \simeq v_{0}\left(n+\frac{1}{2} l+\varepsilon\right)+\delta_{n l}
$$

where

$$
v_{0}=\left(2 \int_{0}^{R} \frac{\mathrm{d} r}{c}\right)^{-1} \simeq 135 \mu \mathrm{Hz}
$$


confirming the uniformly spaced peaks seen in the low $l$ observations (Figure 2). For $n / l \ll 1$, another asymptotic formula gives (see Deubner and Gough, 1984)

$$
\omega^{2} \simeq 2(n+\varepsilon) G,
$$

where $G \equiv \mathrm{d} c^{2} / \mathrm{d} r$ is of order the solar surface gravity. This equation describes parabolas in the $k-\omega$ plane, which correspond to the ridges seen in Figure 1.

The g-modes are distinct from the p-modes, as can be seen in Figure 3, in that they are all trapped below $r=0.75 R_{\odot}$, which is the base of the solar convection zone. This occurs simply because the unstable convecting layers will not support buoyancy oscillations. Asymptotic relations show that low $l$ g-modes, which have the largest surface amplitude for a fixed g-mode energy, have periods which are uniformly spaced, analogous to the uniform frequency spacing of the p-modes. An examination of the
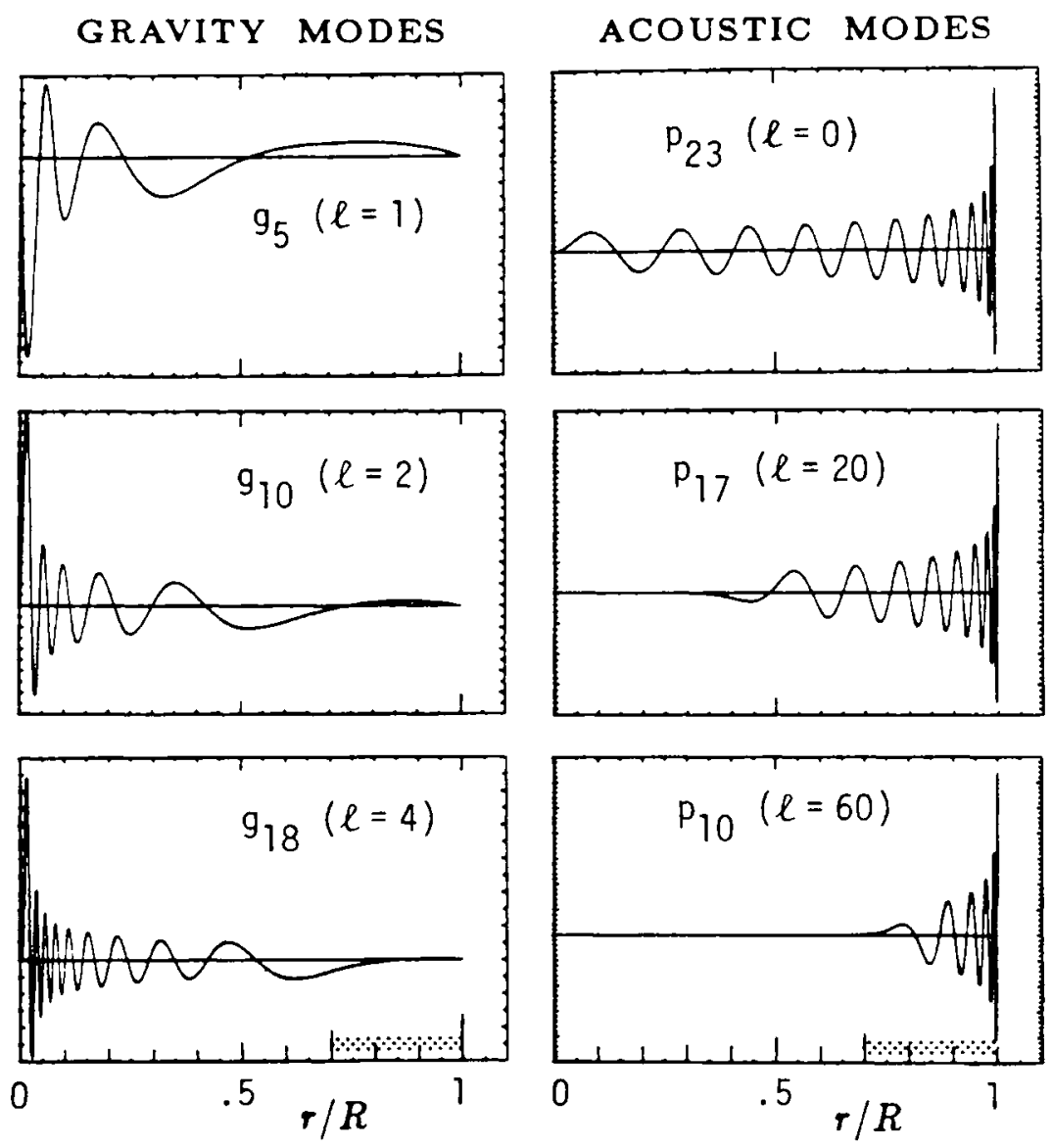

Fig. 5. Vertical component of velocity as obtained from a theoretical solar model for several acoustic and gravity modes of oscillation. Shown is variation with radius within the Sun, scaled by $r \rho^{1 / 2}$, where $\rho(r)$ is density. The three g-modes on the left all have frequencies of about $0.10 \mathrm{mHz}$ (periods of $165 \mathrm{~min}$.); increasing $l$ and $n$ yields modes with more complicated structure near the solar core. The three p-modes have frequencies of about $3.3 \mathrm{mHz}$ (periods of $5 \mathrm{~min}$.); note the p-modes are confined to a region closer to the solar surface as $l$ is increased. The ordinate scales are arbitrary; the horizontal line is at zero. The shaded bands indicate the extent of the convection zone. All the g-modes possess an upper reflection point near the base of the convection zone, and are evanescent within the convection zone. (From Toomre,

1986.) 
g-mode eigenfunctions shows that the mode displacements are predominantly horizontal at the solar surface. The long thermal time-scale of the solar interior suggests that g-mode quality factors should be very high, $Q \sim 10^{12}$ (Press, 1986), giving mode coherence times of $10^{8}$ years.

There is some evidence that g-modes have been observed (see Solar Phys. 83; Delache and Scherrer, 1983). However, these observations have come under doubt as newer observations have not confirmed the earlier detections. Because of the uncertainties in the observations, plus the expectation that g-mode surface amplitudes will be quite small, I will assume here that no g-modes have yet been detected. If g-modes are ever definitely observed, they could provide a great deal of information about the deep interior of the Sun, where the p-modes do not effectively probe. However, the observations promise to be difficult.

The above equation of motion does not describe another class of oscillation modes, the compressionless $(\Phi=0)$ surface waves, referred to as fundamental or f-modes. They are essentially surface gravity waves, with a displacement amplitude proportional to $\exp [-k(R-r)]$, and a dispersion relation $\omega^{2}=g k$. These modes are usually classified with $n=0$. (Although see Deubner and Gough (1984) and Figure 3 in Brown et al. (1986) for a precise definition of the classification scheme.)

Example g- and p-mode eigenfunctions are shown in Figure 5, and calculated eigenfrequencies are shown in Figure 6. Because p-modes are not trapped if their frequencies are above the maximum acoustic cutoff frequency near the solar surface, and the frequencies are monotonically increasing $n$ and $l$, we see that there exists a finite number of p-modes that are trapped inside the Sun, about 10 million. A good fraction of these, those with frequencies between roughly 1 and $5 \mathrm{mHz}$, are excited to observable amplitudes.
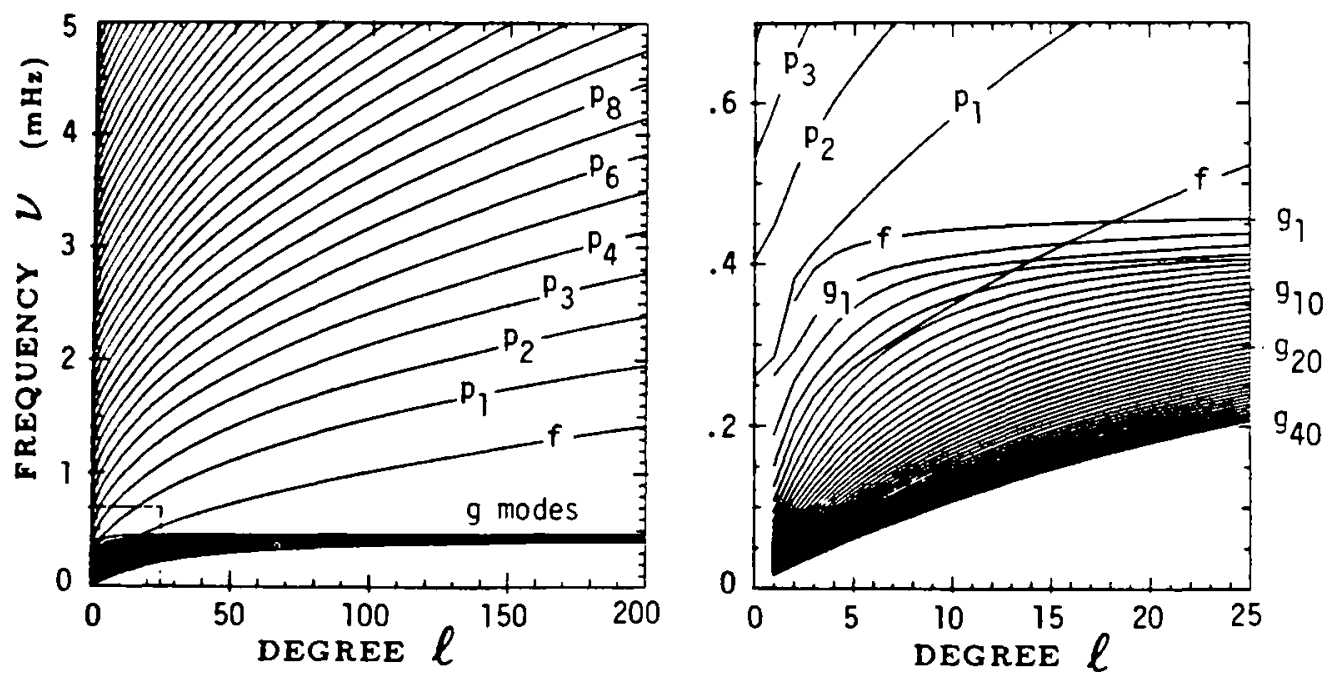

Fig. 6. Eigenfrequencies $v_{n l}$ obtained for p-, g-, and f-modes from a standard solar model; the discrete frequencies of modes of like order $n$ have been connected by lines. The right panel is an expanded view of the left, for low frequencies and degrees. The plotting of g-modes was stopped at $n=40$. (From Toomre, 1986.) 


\section{Observational Techniques}

Two basic techniques have evolved to measure p-mode oscillations on the Sun: Doppler measurements and brightness measurements. The first of these involves accurately measuring the Doppler shift of usually a single spectral line, which has been done using no imaging of the solar surface (i.e., with integrated sunlight), with onedimensional imaging (including after collapsing the solar disk onto a line with a cylindrical lens), and with full 2-D Doppler imaging of the solar disk.

The integrated sunlight measurements are the most difficult of the Doppler measurements, requiring a very stable Doppler analyzer to perform the task. At the peak in the power spectrum, near $3 \mathrm{mHz}$, a single $l=0$ mode has a velocity amplitude in integrated sunlight of only $15 \mathrm{~cm} \mathrm{~s}^{-1}$, requiring a wavelength precision of a part in $10^{10}$ to cleanly detect. To date this feat has only been accomplished using extraordinarily stable atomic resonance cells (Claverie et al., 1979; Grec et al., 1980; Brookes et al., 1978), with which oscillation amplitudes as small as $1 \mathrm{~mm} \mathrm{~s}^{-1}$ have been detected. These measurements are particularly sensitive to transparency fluctuations in the Earth's atmosphere, since the Doppler contributions from the solar limbs $\left( \pm 2 \mathrm{~km} \mathrm{~s}^{-1}\right)$ must precisely cancel if the small net Doppler signal $\left(<1 \mathrm{~m} \mathrm{~s}^{-1}\right)$ from the solar oscillations is to be seen.

In spite of the extreme Doppler stability required by these measurements, they were the first used to observe individual oscillations modes on the Sun, and continue to produce excellent results. The greatest effort with integrated Doppler measurements has been from the Birmingham group (Claverie et al., 1981a, b), using a potassium resonance scattering cell to observe the $7699 \AA \mathrm{K}$ line. A sample day's data from this group is displayed in Figure 7, showing the stability of the resonance scattering

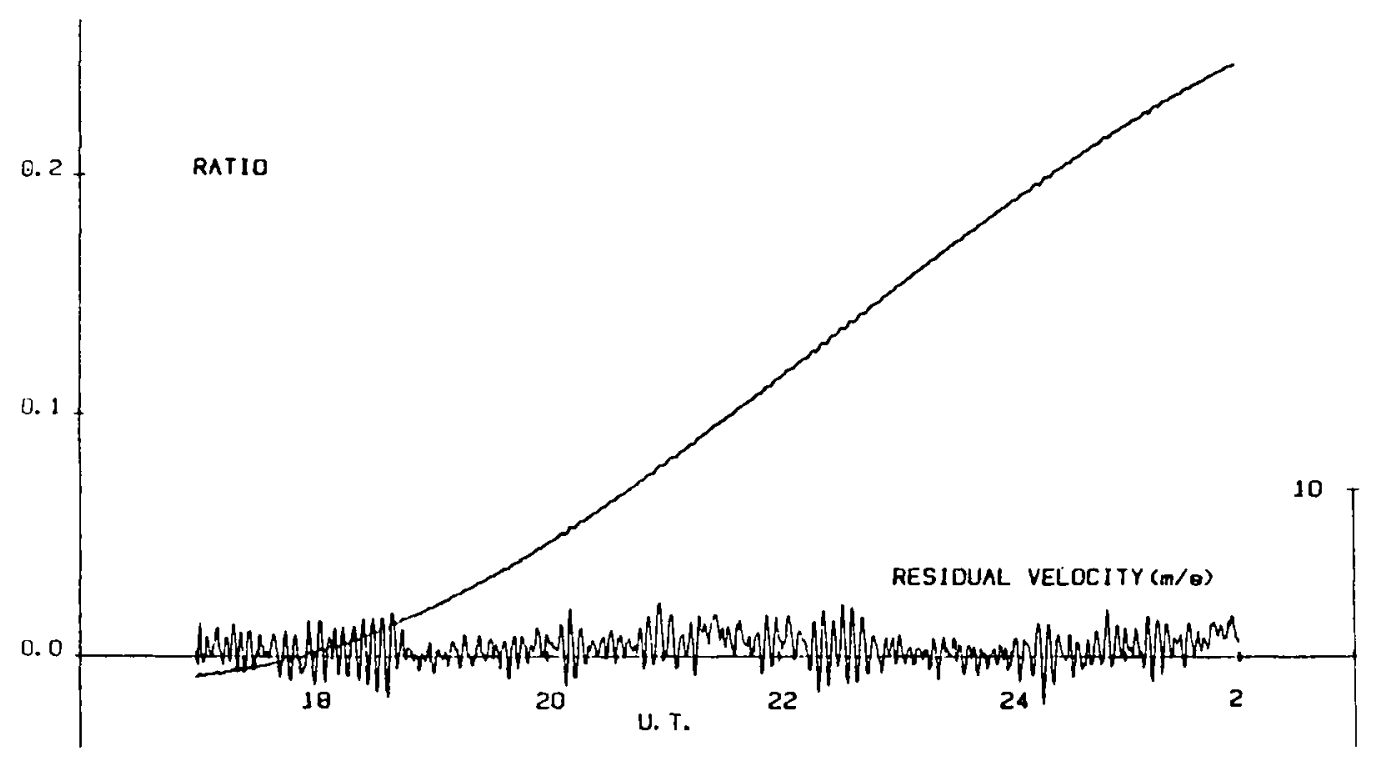

Fig. 7. Diurnal variation of the measured Doppler velocity of integrated sunlight as a function of Universal Time, and velocity residuals after subtracting earth rotation; the latter are normalized to $\mathrm{m} \mathrm{s}^{-1}$, with the scale shown at right. This is a single day's data from Haleakala, Hawaii. (From Isaak, 1986.) 
technique. This group is currently observing from two stations, one at Izana, Tenerife, of the Canary Islands, and the second from Haleakala, Maui, of the Hawaiian Islands. A network of observing stations such as this allows nearly round-the-clock coverage, thereby eliminating some of the problems with temporal sidelobes in the oscillation power spectra (see below).

Two-dimensional Doppler imaging of the sun involves imaging through a narrow bandpass filter which can be chopped or somehow scanned between the red and blue wings of a solar spectral line. In essence a solar image is captured using light from only the blue wing of the line, followed by a second image using the red wing, and the two digital images are subtracted to form a 2-D Doppler image of the Sun, such as is shown by Libbrecht and Zirin (1986). A number of types of narrow bandpass filters have been used for such measurements, including atomic resonance magneto-optical filters (Rhodes et al., 1986; Cacciani and Fofi, 1978), solid Michelson interferometers (called the Fourier Tachometer by its creators; see Brown, 1985), birefringent filters (Libbrecht and Zirin, 1986), and Fabry-Pérot etalons (Rust et al., 1986). By looking at modes with $l \gtrsim 5$, filter drifts with low spatial periods are not a problem, and each of these filters has performed adequately at this task. Although in principle all of these filters could be made stable enough to observe the very low $l$ modes, in practice it has not happened with any of them. The author has noticed that both residual temperature drifts in his filter, as well as low spatial frequency noise in his CCD camera, are to blame for his inability to observe low $l$ modes. It is likely that once these types of problems are cleaned up, the imaging filters will be capable of observing the low-l modes along with the nonimaging devices.

Data collected with imaging Doppler instruments consist of a time-series of solar Doppler images, which is then analyzed in such a way as to isolate as much as possible the individual oscillation modes. This is usually done by first fitting each solar image to some large number (typically $\sim 10^{4}$ ) of projected spherical harmonics $Y_{l}^{m}(\theta, \phi)$, producing a set of fit coefficients $A_{l m}(t)$ at each time point. The fit coefficients are then Fourier transformed in time to produce power spectra. Typical results are shown in Figure 8, which shows power spectra at different $l$ displaced vertically. To improve signal-to-noise, spectra with $-l \leq m \leq l$ were averaged together after removing an estimated rotational splitting (see below). These spectra show some characteristic features of helioseismology data: overlap in $l$, owing to our inability to observe the backside of the Sun (put another way, the spherical harmonics are not orthogonal over half a sphere), and temporal sidelobes at $\pm 11.6 \mu \mathrm{Hz}$, which result from the day/night cycle of the observations. The latter problem can be reduced significantly by combining observations from several stations around the world, while the former is likely to remain a feature for some time to come.

The other technique for observing solar oscillations, through brightness oscillations, is quite simple, involving nothing more than an imaging or non-imaging device which observes the brightness of the solar surface as a function of time, over a wavelength interval determined by a bandpass filter. Brightness oscillations in integrated sunlight are very small, of order $10^{-6}$ in the continuum, and are too small to observe from the 


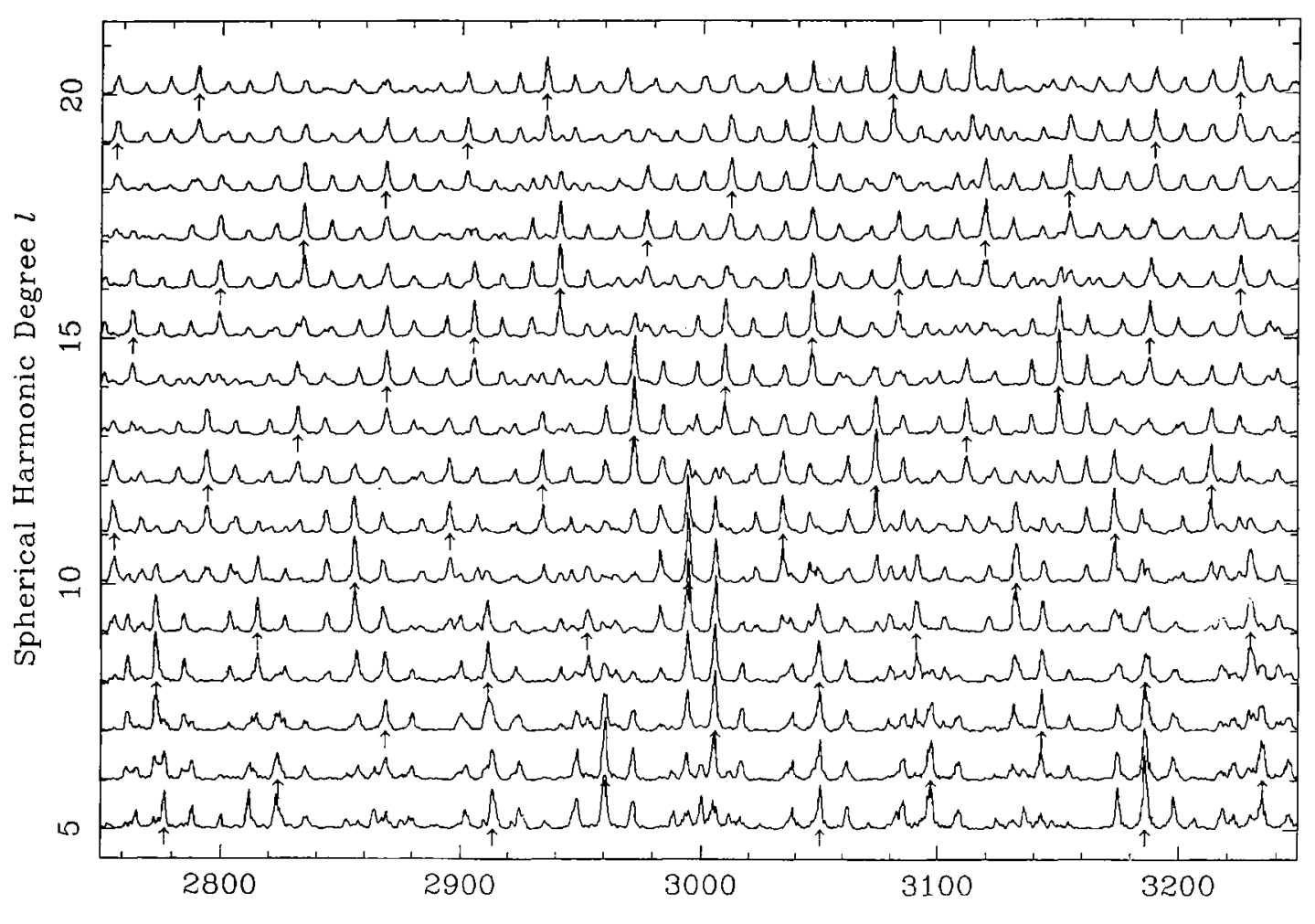

Frequency, $\mu \mathrm{Hz}$

Fig. 8. Power spectra of $Y_{l}^{m}$ fit coefficients for each $l$ in the range $5 \leq l \leq 20$, plotted over a small range in frequencies; these are in fact short vertical cuts through the power diagram shown in Figure 1. Each spectrum at a given $l$ is an average of $2 l+1$ spectra with all values of $m$ for that $l$, after first shifting the spectra in frequency to correct for rotational splitting. Identified solar mode frequencies $v_{n l}$ are marked with vertical arrows below each spectrum. Crosstalk between the different $l$ results from observing only one side of the Sun, and temporal sidelobes at $\pm 11.6 \mu \mathrm{Hz}$ appear because of the day/night observing window. Note that virtually every feature can be explained as a true oscillation mode or an alias of one, and that the aliases are always smaller than the true peaks. (From Libbrecht and Zirin, 1986.)

ground. These were first observed in with the Active Cavity Radiometer Irradiance Monitor (ACRIM) experiment aboard the Solar Maximum Mission satellite (Woodard and Hudson, 1983, 1984), which observed for 290 days in 1980 before the loss of spacecraft fine pointing control. The power spectrum of the brightness fluctuations is similar in appearance to Figure 2. At the 5-min peak of the oscillation spectrum, the ratio of brightness to velocity oscillation of a single mode is roughly

$$
\frac{(\Delta I / I)^{2}}{v^{2}\left(\mathrm{~cm}^{2} \mathrm{~s}^{-2}\right)} \simeq 1.5 \times 10^{-14}
$$

In 1984 the spacecraft was repaired in orbit by the Space Shuttle, and observations have been underway since that time.

Measurements of brightness oscillations using imaging detectors have been done from the ground, again detecting only the higher $l$ modes. Broadband measurements suffer from low signal-to-noise (Nishikawa et al., 1986), and are, therefore, unsuited for 
frequency measurements, but measurements using a narrow bandpass filter centered on the Ca K line have worked out quite well (Duvall et al., 1988), since the oscillation signal $\Delta I / I$ is considerably stronger in a deep spectral line.

Since brightness oscillations are not described by the simple adiabatic theory, interpretation of the brightness oscillation amplitudes from a solar atmosphere model, knowing the velocity amplitudes, presents an interesting challenge. This aspect of the field will probably be explored more carefully in the next few years, and will perhaps teach us more about the structure of the solar atmosphere.

\section{P-Mode Frequencies}

Because the p-modes penetrate deep into the Sun, their frequencies naturally depend on the properties of the solar interior. Therefore, the p-modes can be used as a probe of the solar interior, much as seismic waves in the Earth can be used to probe the Earth's interior. In the past, theoretical solar models were constrained to reproduce little other than the measured mass, radius, and luminosity of the Sun. Starting with a solar model containing the correct solar mass, the latter two quantities could be matched by a judicious adjustment of the mixing-length parameter and the initial solar helium

\section{Solar P-Mode Frequencies, Data and Theory}

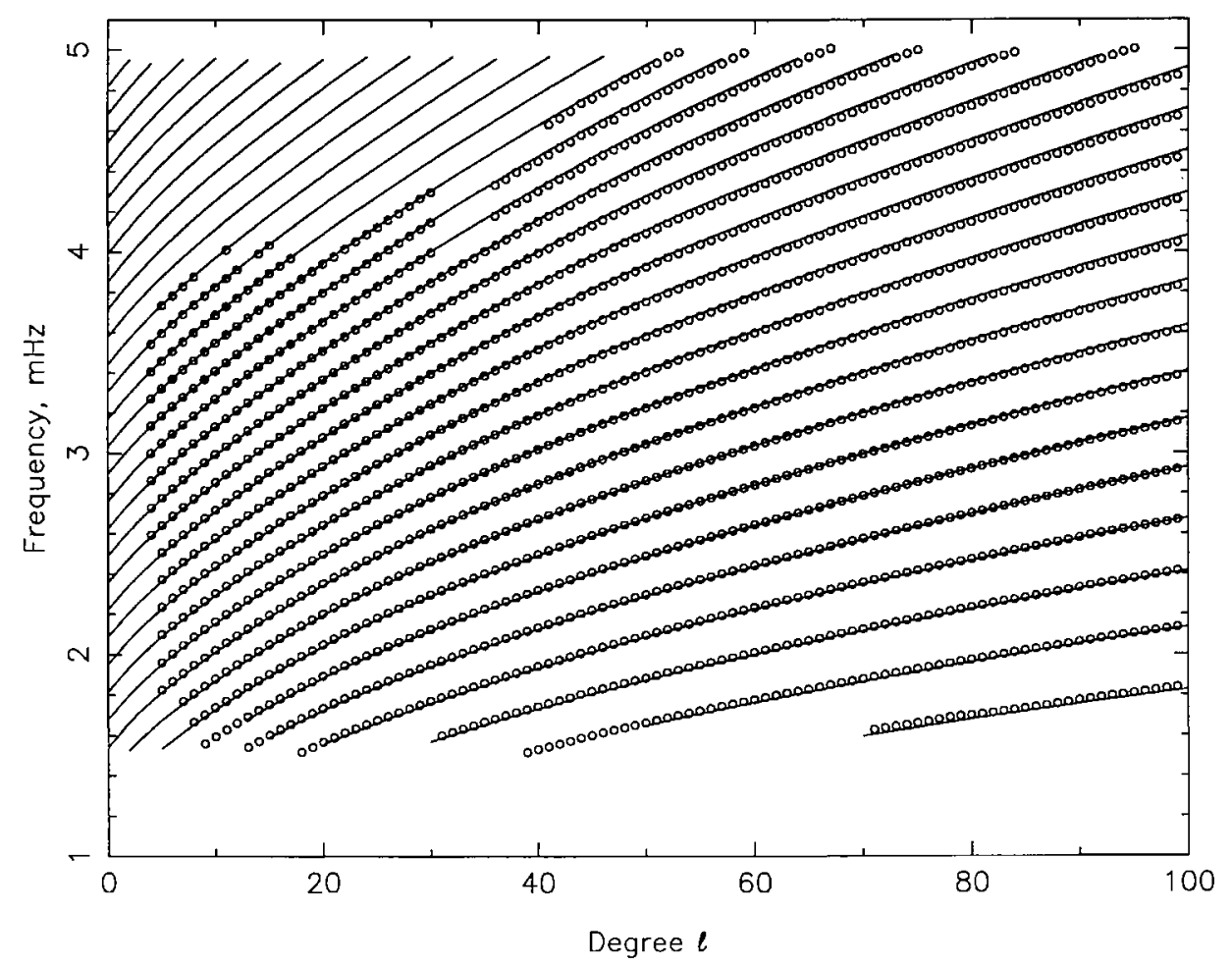

Fig. 9. Measured solar p-mode frequences $v_{n l}$ from Duvall et al. (1988) (circles), along with frequencies calculated from a standard solar model by Christensen-Dalsgaard. The theoretical points with fixed $n$ have been connected by lines. Uncertainties in the measured frequencies are typically one part in $10^{4}$ in the center of the figure. The short ridge in the lower right has $n=2$, and $n$ increases by one for each higher frequency ridge. 
abundance, both of which are very poorly known from independent measurements. The new and very accurate observations of $\mathrm{p}$-mode frequencies provide thousands of additional, independent constraints for the solar model calculations. Using these new-found model constraints to learn more about the solar interior structure is currently a very active branch of helioseismology.

The input data for these studies is simply a table of $v_{n l m}$, and the most recent measurements by Duvall et al. (1988) and Libbrecht and Kaufman (1988) are the best so far. Frequencies measured by these authors are shown for $l \leq 100$ in Figure 9, along with a model calculation by Christensen-Dalsgaard (1982). The first thing to notice from this figure is the very small amount of scatter in the measured frequencies; the measured frequency uncertainty for most of the points is a few tenths of a $\mu \mathrm{Hz}$, or a part in $10^{4}$. With these new measurements, solar p-mode frequencies are now among the most accurately determined physically significant quantities in all of astrophysics. (For contrast, pulsar period measurements have been made to accuracies of $10^{12}$ or greater, but this reflects simply a rotation rate of a neutron star, which returns no physical understanding of the phenomenon. Pulsar period derivatives are physically interesting, but their measurements are not nearly so precise, to a part in $10^{4}$ with the binary pulsar $1913+16$, for example.)

A second thing to notice from Figure 9 is the fairly good agreement between the measured and calculated frequencies. While the agreement is significantly worse for

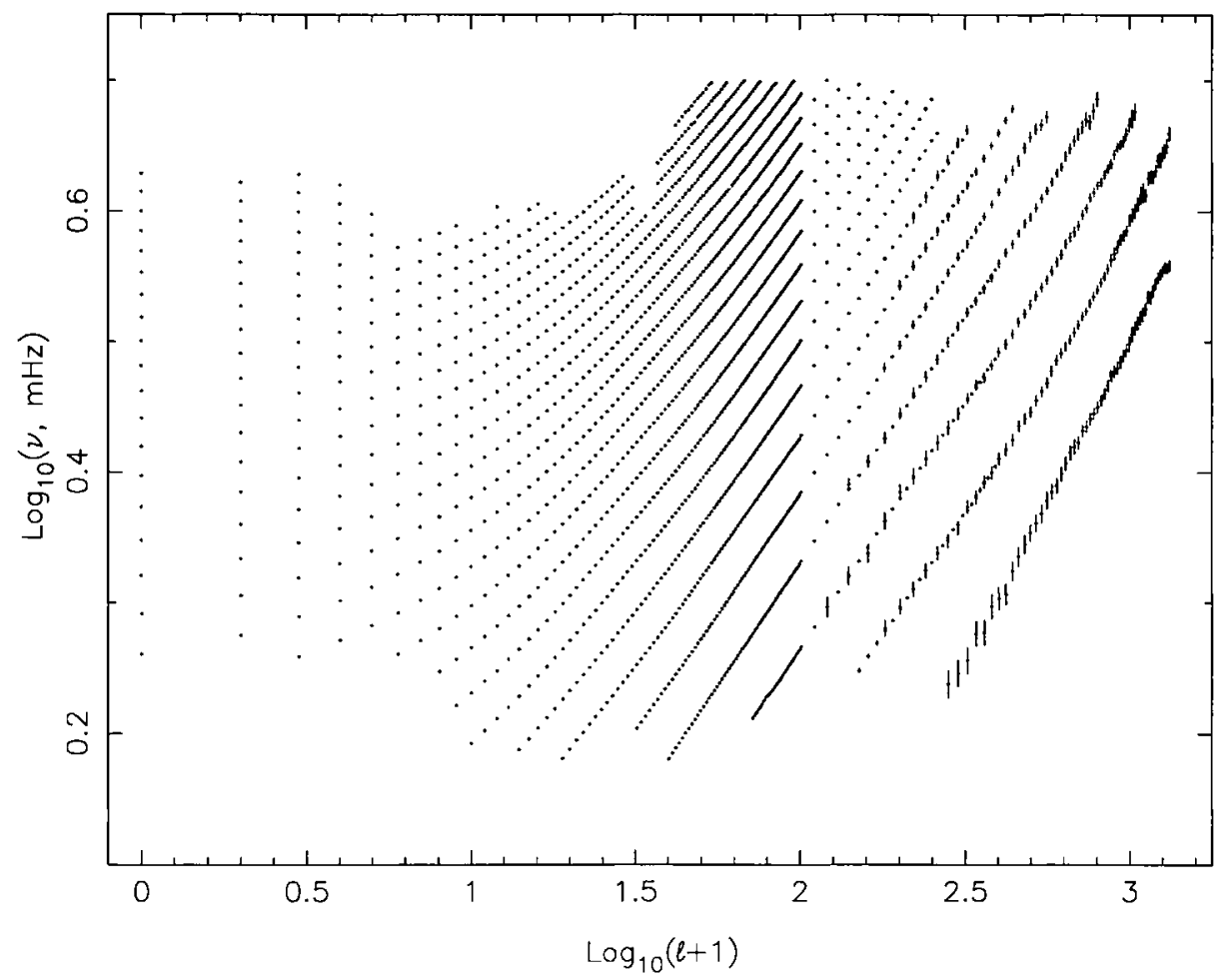

Fig. 10. Measured solar oscillation frequencies, from Duvall et al. (1988) and Libbrecht and Kaufman (1988). The line of frequencies at the lower right is the f-mode ridge, which fits the dispersion relation $\omega^{2}=g k$, with $\omega=2 \pi v$ and $k \simeq l / R_{\odot}$. 
higher $l$-values, it nevertheless proves our basic understanding of the p-modes as trapped acoustic oscillations described by adiabatic perturbations of a standard solar model.

Measurements at higher $l$ values are included in Figure 10, showing the rather greater measurement uncertainties in this region. Note the lowest frequency ridge at high $l$, which consists of the f-modes, which to the accuracy of the measurements fit the theoretical dispersion relation above, $\omega^{2}=g k$.

Using the p-mode frequencies to determine the solar interior structure is not a straightforward task, and two approaches are generally followed. The first is to pursue the forward problem, or in other words to calculate p-mode frequencies from a bevy of evolved solar models, each with slightly different interior properties, and see which best fits the data. This approach is especially useful for determining which model uncertainties are important in the calculation of the p-mode frequencies.

The forward problem has been explored by a number of authors, working primarily with the very accurate low $l$ frequency measurements that came out the of the integrated sunlight Doppler observations. Christensen-Dalsgaard (1982) computed eigenfrequencies from a number of solar models, and found numerical errors (using 200 radial mesh points) were of order $\Delta v / v \sim 10^{-3}$, while frequency differences from using two different opacity tables resulted in an error estimate of $\sim 3 \times 10^{-3}$. Ulrich and Rhodes (1983) concluded with their solar model that errors in the calculated low $l$ frequencies from interior physics, including uncertainties in nuclear cross sections, the equation of state, and the opacity table were of order $3 \times 10^{-4}$, whereas uncertainties in the outer boundary condition could introduce errors as large as $3 \times 10^{-3}$. Nonstandard solar models were also investigated by Ulrich and Rhodes. Shibahashi et al. (1983) found that electrostatic corrections to the equation of state produced a frequency change of $3 \times 10^{-3}$ for the low $l$ modes, giving a considerably better agreement with the measured frequencies than without the correction. However, Noels et al. (1984) obtained different results using similar techniques, for reasons that are not altogether clear. Faulkner et al. (1986), and independently Däppen et al. (1986), found that a better fit to the low $l$ data could be gotten by adding weakly interacting massive particles (WIMPs) to the solar core, which also alleviated the solar neutrino problem. The effects of various model parameters on high $l$ frequency calculations was explored by Lubow et al. (1980). Brown (1984) investigated the effects of convection on p-mode frequencies. None of the results above is close to being considered the final word on this topic, as indeed none of the calculations was able to reproduce the low $l$ observations to within experimental error. It is hoped that the new frequency measurements covering a large range in $l$ will spur further exploration of the forward problem. It remains to be seen just what model improvements will be necessary to finally fit the data.

The second approach to inferring the interior properties of the Sun from the p-modes is through an inversion of the mode frequencies to determine the speed of sound throughout the solar interior directly. A first attempt at this formidable inversion was presented by Christensen-Dalsgaard et al. (1985), using an idea by Duvall (1982) for reducing the $\mathrm{p}$-mode frequency data to a more manageable form.

Duvall noticed that the outer or surface boundary for p-modes is roughly independent 
of $l$ and mode frequency over the small frequency range of the observations, while the inner boundary $r_{i n}$ is given by $\omega=c\left(r_{i n}\right) k_{h}$, where $k_{h}$ is the horizontal wave number $k_{h}=\sqrt{l(l+1)} / r$, and $c(r)$ is the sound speed inside the Sun. Thus modes with similar $\omega / k_{h}$ should be confined to the same solar cavity, and the sound travel time across the cavity, given approximately by $n \pi / \omega$, should be a function of the cavity, i.e., $n / \omega=f\left(\omega / k_{h}\right)$. A modified version of this relation, $(n+\alpha) / \omega=f\left(\omega / k_{h}\right)$, with $\alpha \simeq \frac{3}{2}$, was more closely satisfied by the data, which is shown in Figure 11. Note that this collapse of the $k-\omega$ diagram to a single ridge shows simply that a single function, the sound speed $c(r)$ as a function of depth inside the Sun, essentially determines the frequencies of all the p-modes.

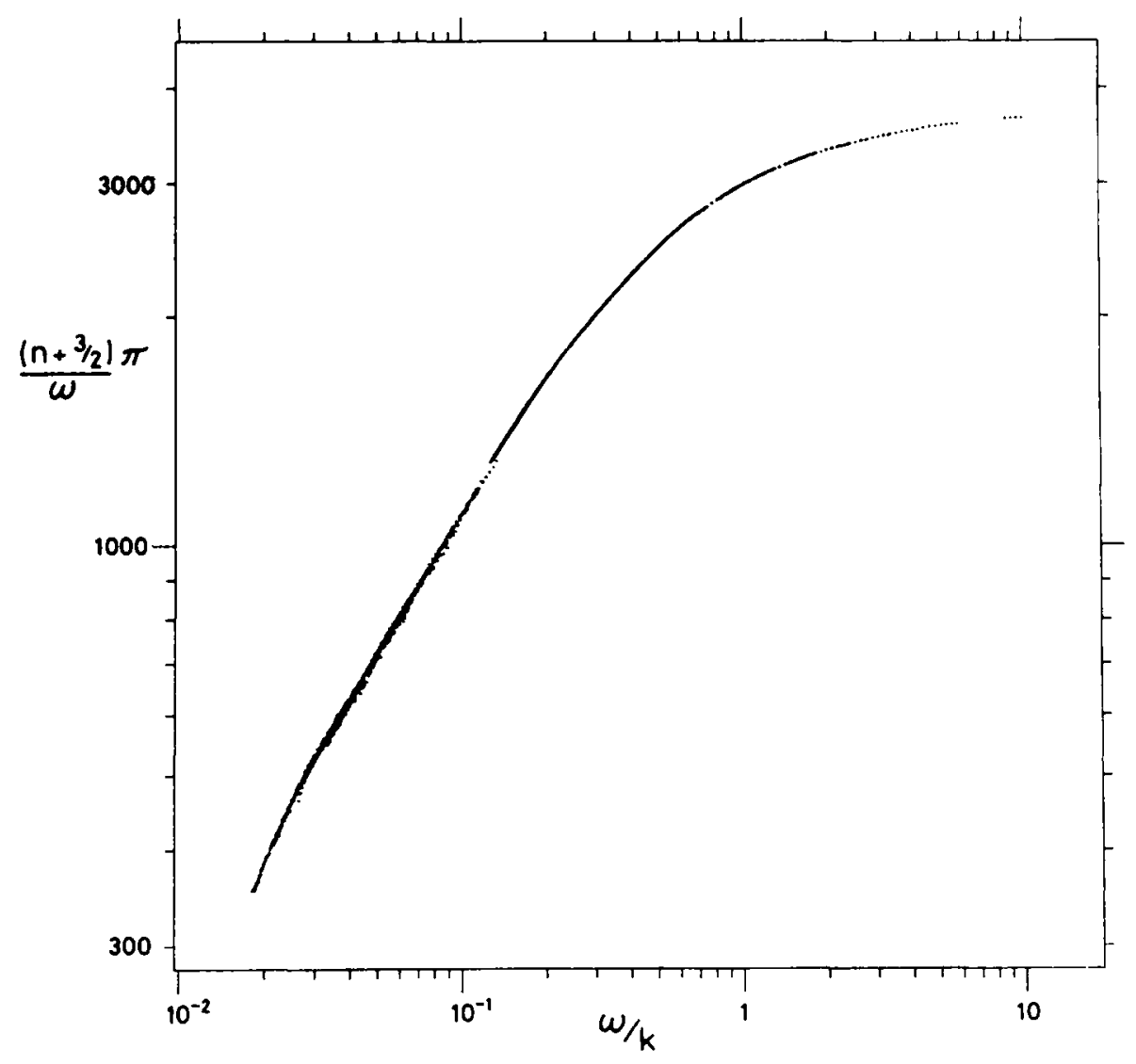

Fig. 11. Representation, in the manner of Duvall (1982) described in the text, of nearly 3000 p-mode frequencies. (From Deubner and Gough, 1984.)

The relation of Duvall was inverted by Christensen-Dalsgaard et al. to determine $c(r)$ directly from the data, and the result, shown in Figure 12, was found to agree fairly well with the standard solar model. Note the inverted $c(r)$ shows a change in curvature at $0.7 R_{\odot}$, marking the base of the convection zone. Although the Schwarzschild criterion has long fixed the radius of the onset of convection in the Sun's interior, this radius has now for the first time been independently measured using the p-mode frequencies. 


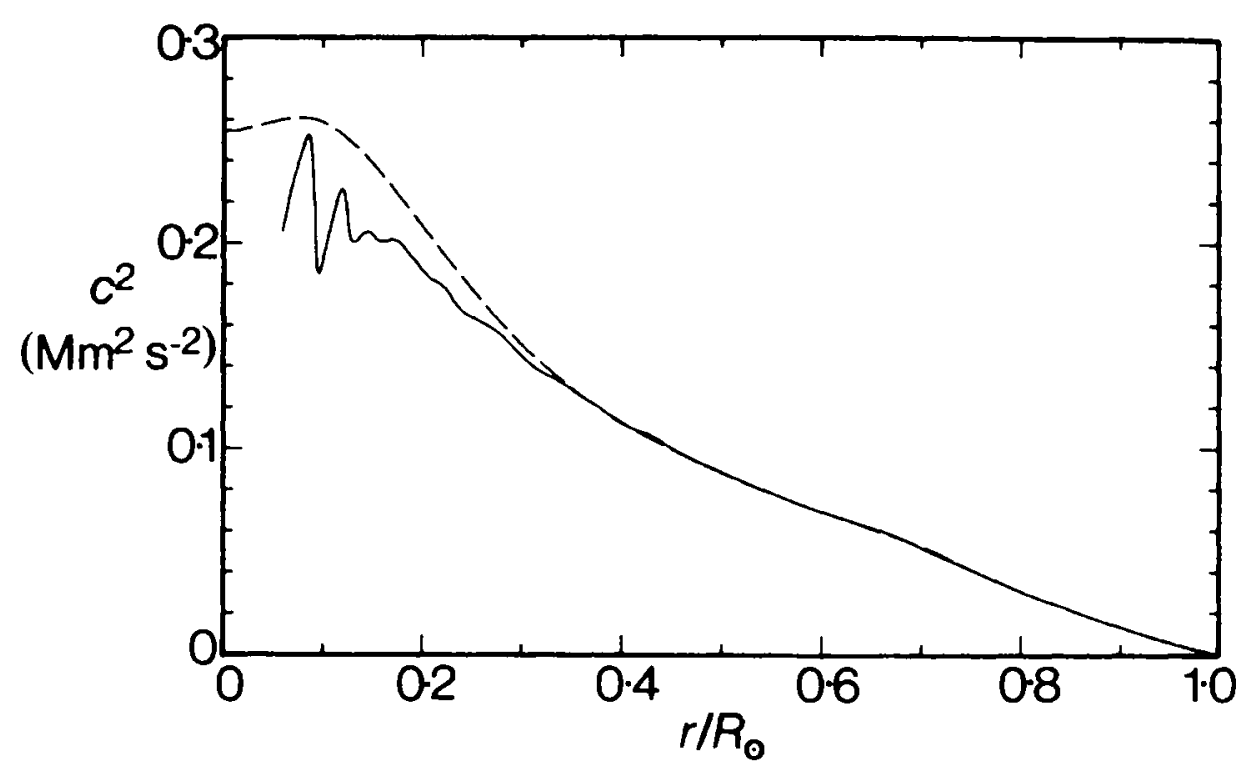

Fig.12. Square of the sound speed inside the Sun, obtained by Christensen-Dalsgaard et al. from the inversion of p-mode frequency data. The dashed line is from a standard solar model, for reference. Comparison of this inversion with the inversion of p-mode frequencies calculated from the solar model showed a discrepancy in the squared sound speed between data and model of less than 3 percent throughout the solar interior with $r>0.15 R_{\odot}$. (From Christensen-Dalsgaard et al., 1985)

Unfortunately, frequency measurements that are free of systematic errors at the tenth of a percent level have only been available for a large range of $l$ for a short time. The recent work by Duvall et al. (1988) and Libbrecht and Kaufman (1988) is almost an order of magnitude improvement over previous measurements. Thus we must wait to see, after the theoretical dust settles, what important deductions can be made about the solar interior from the oscillation frequencies.

\section{Rotational Splittings and Frequency Shifts}

For a truly spherical Sun, the p-mode frequencies $v_{n l m}$ would be degenerate in $m$. Because the Sun is rotating, however, this degeneracy is lifted, and multiplets with a given $n$ and $l$ are said to show a rotational splitting of the frequencies of the different $m$ states, analogous to the Zeeman splitting of the energy levels of an atom. Convection in the Sun brings about a solar rotation rate which is not constant as a function of depth and latitude. While the surface rotation rate as a function of latitude has been measured using sunspot motion and Doppler shifts (Howard, 1984), the rotation rate as a function of radius was, until recently, largely unknown. It has been suggested that the deep interior of the Sun has decoupled from the surface layers, and may be rotating substantially faster than is observed at the surface (Dicke, 1970). Therefore, an effort is now underway to use the measured rotational splitting of p-modes to directly measure the interior rotation rate of the Sun.

For a uniformly rotating Sun, the rotational splitting of the p-modes is very roughly 
given by

$$
v_{n l m}=v_{n l 0}+m v_{\odot},
$$

where $v_{\odot}$ is the rotation frequency of the Sun, which is approximately $0.4 \mu \mathrm{Hz}$. Including solar differential rotation as a function of depth and latitude, the splitting is more complicated and has been calculated for the case when centrifugal effects can be ignored by Cuypers (1980) and Gough (1981). Latitudinal differential rotation introduces small $m^{3}$ and higher order terms into the splitting, although for no form of rotation $v_{\odot}(r, \theta)$ does one expect a splitting described by even powers of $m$ (in this approximation, where the solar oblateness is negligible). Differential rotation as a function of depth shows up most clearly as an $l$-dependence in the measured splitting, resulting from the fact that the inner turning point of the p-modes depends strongly on $l$.

It has become customary to fit the observed frequencies $v_{n l m}$ to Legendre polynomials in $m / l$ :

$$
v_{n l m}-v_{n l 0} \rightarrow l \sum_{i=1}^{N} a_{i} P_{i}(-m / l)
$$

producing in principle a set of fit coefficients $a_{i}$ for each multiplet of a given $n$ and $l$.
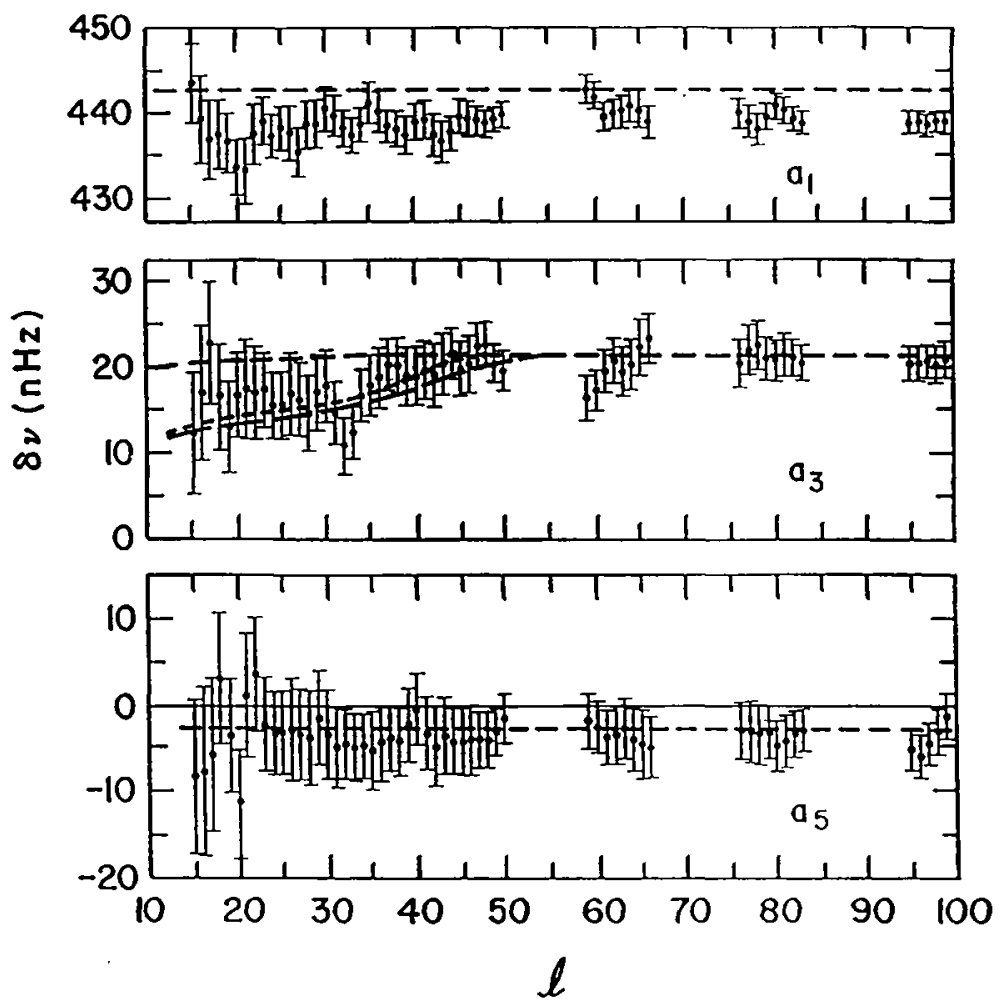

Fig. 13. Frequency splitting coefficients $a_{i}$ for $i=1,3,5$, in sidereal $\mathrm{nHz}$, measured by Brown and Morrow (1987). The coefficients here have been averaged over $n$; even $i$ coefficients appear to be consistent with zero. Dashed lines indicate the values one would expect if the surface differential rotation prevailed at all solar radii, while the other curves are for different assumptions about the interior rotation rate. (See Brown and Morrow for details). 
The observed surface rotation, as measured by following magnetic features, yields coefficients (in $\mathrm{nHz}$, sidereal) of $a_{1}=442.8, a_{3}=21.7$, and $a_{5}=-2.5$ (Brown and Morrow, 1987). An estimate of the effect of the finite solar oblateness gives (Gough and Taylor, 1984) $\Delta v / v \simeq 0.3 \Delta R / R$, where $\Delta v=v_{n l l}-v_{n l 0}$ and $\Delta R=R_{\text {equator }}-R_{\text {pole }}$, which then gives $a_{2} \simeq 5 l^{-1} \mathrm{nHz}$.

The most recent published data by Brown and Morrow (1987) is shown in Figure 13, along with splittings calculated from a standard model with several estimated rotation curves. Note there are some minor discrepancies between this data set and another by Duvall et al. (1986), including a significantly non-zero $a_{2}$ in the latter set; future observations should clear up these differences. The only firm result to date is that the solar rotation rate in the equatorial regions decreases slowly with depth throughout the convection zone. Full theoretical inversions to infer $v_{\odot}(r, \theta)$ in the convection zone, and hopefully into the core region as well, are only now being realized.

There have also been reports of p-mode frequency shifts with time, presumably due to the solar cycle, such as from Van der Raay (1984), Woodard and Noyes (1985), and Duvall et al. (1988). The results do not all agree with one another, so it is not yet clear whether a real effect has been seen. However, one can say from the existing measurements that any solar cycle p-mode frequency shift are at most $\sim 1 \mu \mathrm{Hz}$ over the cycle. Bogdan and Zweibel (1985) investigated frequency shifts resulting from fibral magnetic fields, and calculated shifts of a few $\mu \mathrm{Hz}$ for realistic field configurations.

\section{Mode Excitation}

While the frequencies of the Sun's p-modes are fairly well understood in terms of adiabatic oscillations, and can be calculated to an accuracy of a percent or so with the standard solar model, we must also ask what it is that excites the modes to their observed amplitudes. Just as the observed mode frequencies can be used to infer certain aspects of the Sun's interior structure, such as the interior rotation rate, the observed mode amplitudes and linewidths can be used to learn about the dynamics of the solar interior that drives the modes.

The first thing one notices about the Sun's p-mode oscillations is that they are qualitatively different than oscillations that have been observed in other stars. While even in integrated sunlight many solar oscillation modes can be observed (Figure 2), other oscillating stars, such as the Cepheid and RR Lyrae variables, tend to show only one or occasionally a few different modes that are simultaneously excited.

Looking at the p-mode data, we see that the velocity amplitudes are peaked around $3 \mathrm{mHz}$, with very little power observed outside the $2-4 \mathrm{mHz}$ range, a result which is true for all $l$ values. Using calculated eigenfunctions we can convert amplitudes to a mean energy per oscillation mode, as is shown in Figure 14. Here we see that the energy per mode is also peaked at $3 \mathrm{mHz}$, but appears to level off to a constant value at low frequency, while falling to zero at the highest frequencies. This energy curve is roughly independent of $l$, at least out to $l \sim 200$. For modes with $l \lesssim 100$, we find that the coherence lifetime of an individual mode (as measured by the linewidth) is a strong 
function of frequency, ranging from less than a day at $5 \mathrm{mHz}$ to over a month at $1.5 \mathrm{mHz}$ (Libbrecht, 1988). Put another way, the quality factor $Q$ for the p-mode oscillations ranges from $Q \sim 100$ at $5 \mathrm{mHz}$ to $Q \gtrsim 5000$ at $1.5 \mathrm{mHz}$.

\section{Solar P-Mode Energies}

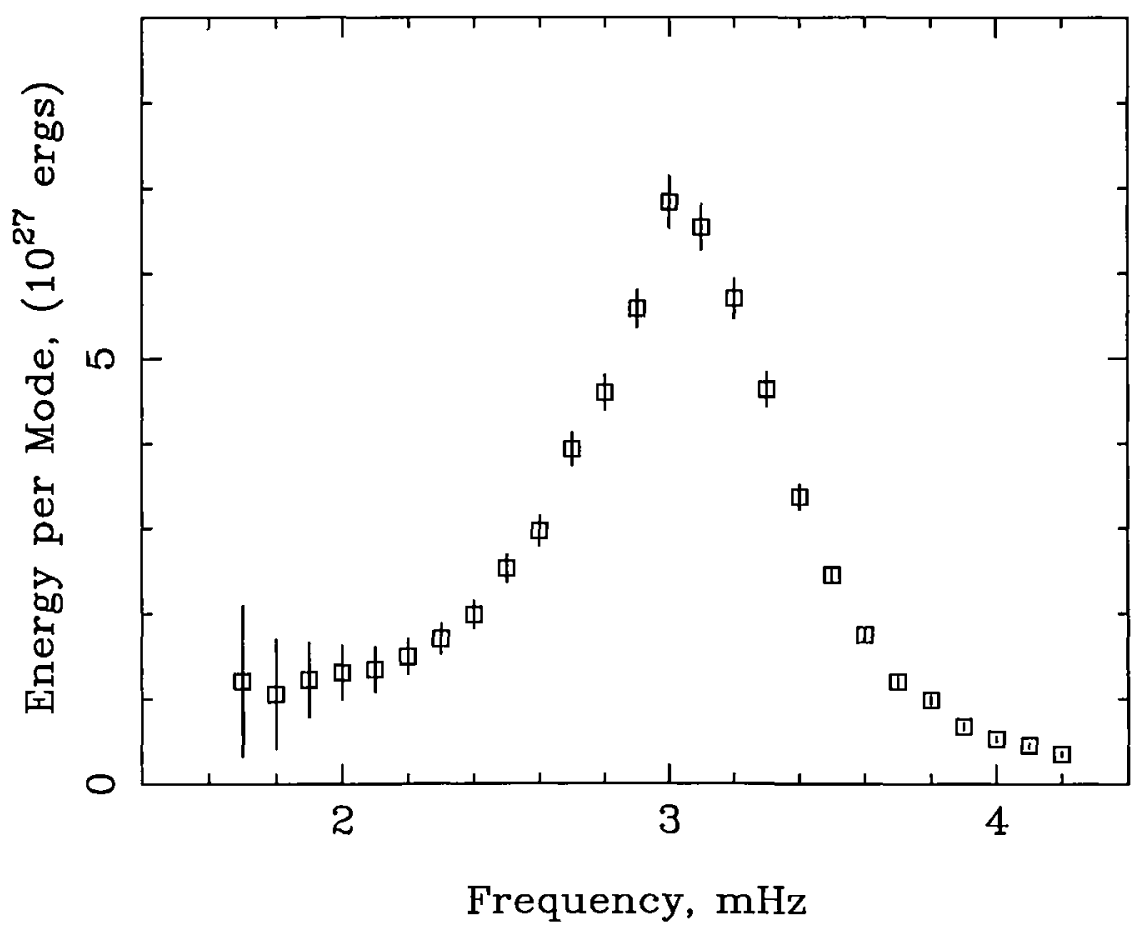

Fig. 14. Energy $E_{n l m}$ per mode of solar p-mode oscillations, measured for low $l$ modes as a function of frequency. The overall normalization of this curve could be in error by as much as $25 \%$ (Adapted from Libbrecht et al., 1986, but using improved data.)

Two possible excitation mechanisms have been discussed extensively in the literature. The first is that the modes are overstable and, therefore, self-excited via the $\kappa$ mechanism, which is the mechanism known to be responsible for the oscillations of Cepheid and RR Lyrae stars. A number of authors have attempted to calculate the linear stability of the p-modes (Ando and Osaki, 1975; Goldreich and Keeley, 1977a; Christensen-Dalsgaard and Frandsen, 1983; Kidman and Cox, 1984; Antia et al., 1986), with mixed results. Theoretical uncertainties in the interaction of the oscillations with radiation as well as convection appear to make it impossible at present to definitely say whether the modes are stably damped or overstable. This situation may change in the future as the calculations become more sophisticated.

If one simply assumes that the modes are in fact overstable, then linear theory describes an exponential growth in the mode amplitudes, until some non-linear mechanism takes over to limit this growth to the observed amplitudes. No mechanism 
has been proposed which could damp uncoupled p-modes, since their amplitudes are much too small for any known nonlinear mechanism to be important. The maximum Mach number $M=v / c$ for the most energetic p-modes occurs near the upper boundary, and is of order $\left(20 \mathrm{~cm} \mathrm{~s}^{-1}\right) /\left(10 \mathrm{~km} \mathrm{~s}^{-1}\right)=2 \times 10^{-5}$. However, if the modes are strongly coupled, then the observed surface amplitude from all the methods $(M \simeq 0.1)$ could perhaps be sufficient to damp the modes. This possibility has not yet been fully explored.

One observation that can be made from the existing stability calculations is that none of the calculations predicts overstability for the f-modes, which are observed to have amplitudes that are roughly in accord with an extrapolation of p-mode amplitudes to higher $l$ and lower $v$. This is not surprising, since the $\kappa$ mechanism works through compression of the solar plasma, and the f-modes are to first-order compressionless. Thus if the $\kappa$ mechanism is responsible for exciting the p-modes, some form of mode coupling must be responsible for transfering energy into the f-modes. However, a recent calculation by Kumar and Goldreich (1988) has shown that the strongest form of coupling, 3-mode coupling, will drain energy from, rather than supply energy to, the f-modes. Since this leaves no means of driving the f-modes, we reach the conclusion that the $\kappa$ mechanism is probably not the source of $\mathrm{p}$ - and $\mathrm{f}$-mode excitation.

An alternative excitation mechanism is stochastic excitation by turbulent convection, which was originally proposed by Goldreich and Keeley (1977b), and later expanded by Goldreich and Kumar (1988). Essentially, the acoustic noise generated by turbulent motions in the convection zone is trapped inside the Sun, which results in the excitation of the Sun's normal modes.

The emission of acoustic noise by turbulent fluids was first theoretically described by Lighthill $(1952,1954)$, and these ideas were expanded by the above authors to include both the emission and absorption of acoustic radiation in a closed box of turbulent fluid. The full theory is quite subtle, and the reader is referred to Goldreich and Kumar (1988) for a full discussion. However, the result is quite simple: for acoustic modes in equilibrium with turbulent convection, the energy per mode is given approximately by $E \sim m c^{2}$, where $m$ is the typical mass of a convective eddy whose turnover time is equal to the oscillation period, and $c$ is the sound speed inside the eddy.

For 5-min oscillations, a resonant convective eddy is simply a solar granule, for which we write $E=\rho H L^{2} \mathrm{c}^{2}$, where $\rho \sim 10^{-7} \mathrm{~g} \mathrm{~cm}^{-3}$ is the density at $\tau_{5000}=1, H \simeq 100 \mathrm{~km}$ is the scale height, $L \sim 1000 \mathrm{~km}$ is the horizontal size of a granule, and $c \sim 10 \mathrm{~km} \mathrm{~s}^{-1}$, given $E \sim 10^{28} \mathrm{ergs}$, in rough agreement with the observations. Note that the turbulent excitation model not only predicts the correct order of magnitude for the p-mode energies, but it also naturally explains the observation that many millions of modes are excited to nearly the same energy.

If we assume this model is essentially correct, then we can qualitatively explain all the features of the observed oscillation power spectra. Figure 15 shows measured velocity mode amplitudes, with the familiar strong peak at a period of $5 \mathrm{~min}$. At low frequencies, below $2 \mathrm{mHz}$, the mode energy levels off to roughly a constant value. However, the mode mass increases sharply with decreasing frequency, as shown in Figure 16, explaining the sharp drop in observed power at low frequencies. 


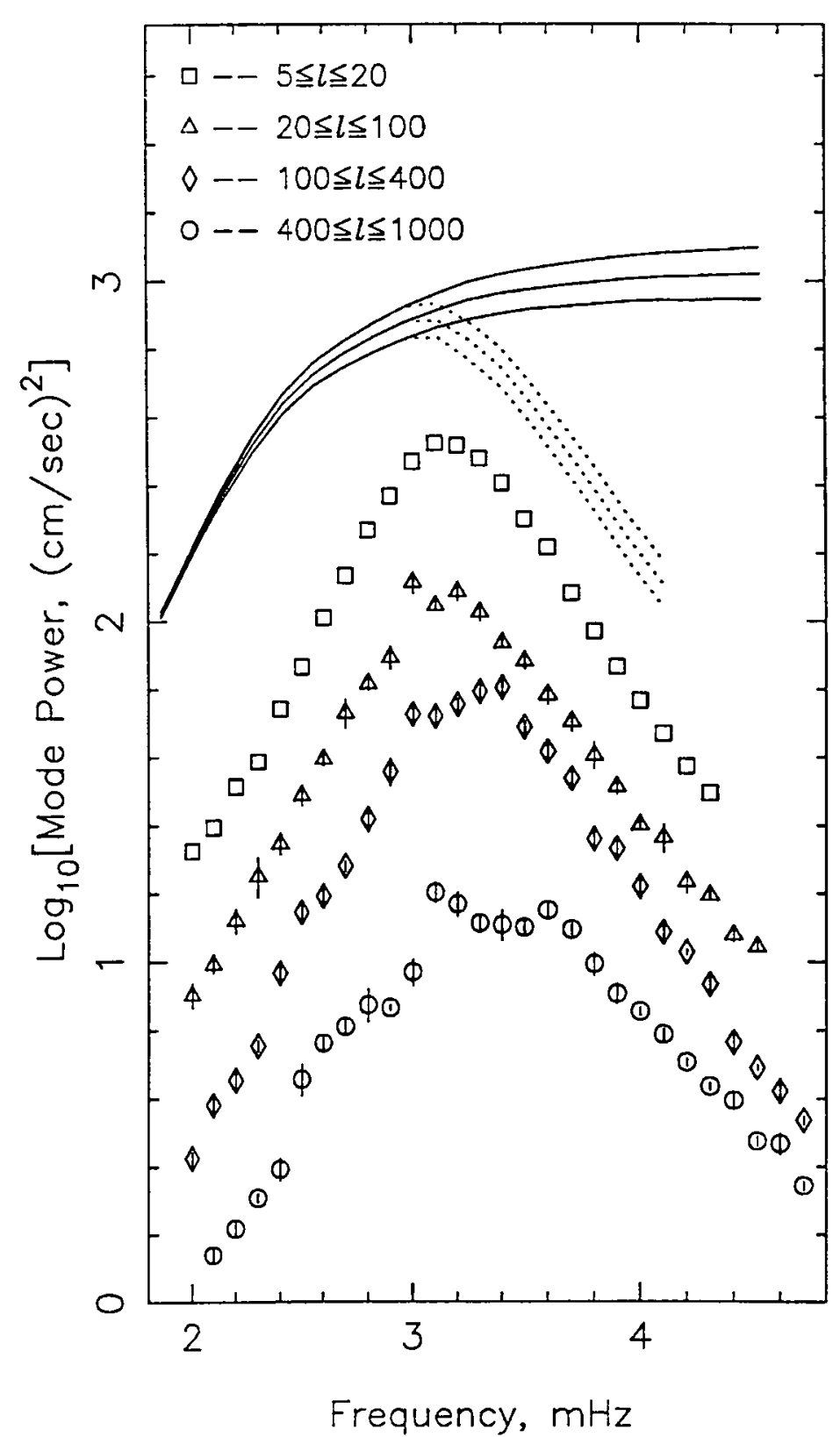

Fig. 15. Measured mode velocity power $P_{v}$, in $\mathrm{cm}^{2} \mathrm{~s}^{-2}$, as a function of frequency, for four different ranges in $l$. Only the lowest $l$ data are properly normalized; the other three sets of points were arbitrarily scaled (see Libbrecht et al. (1986) for the power as a function of $l$ ). The solid lines give the scaled logarithm of the inverse mode mass (28- $\log _{10} M$ ), where $M$ is the calculated mode mass shown in Figure 16; this is the expected functional form of $P_{v}$ if the modes all have equal energies. The upper, middle, and lower curves were calculated for optical depths $\tau_{5000}=0.02,0.05$, and 0.1 , respectively, with $l=10$. The dotted lines are [28- $\log _{10}(M \Gamma)$ ), where $\Gamma$ is the measured mode linewidth in $\mu \mathrm{Hz}$. (From Libbrecht et al., 1986.)

At lower frequencies the modes are trapped deeper inside the Sun, and so it is likely that the only significant damping mechanism at work on the oscillations is the exchange of energy with turbulent convection. At higher frequencies, radiative damping becomes the dominant drain of energy from the modes. This is because at higher frequencies the 


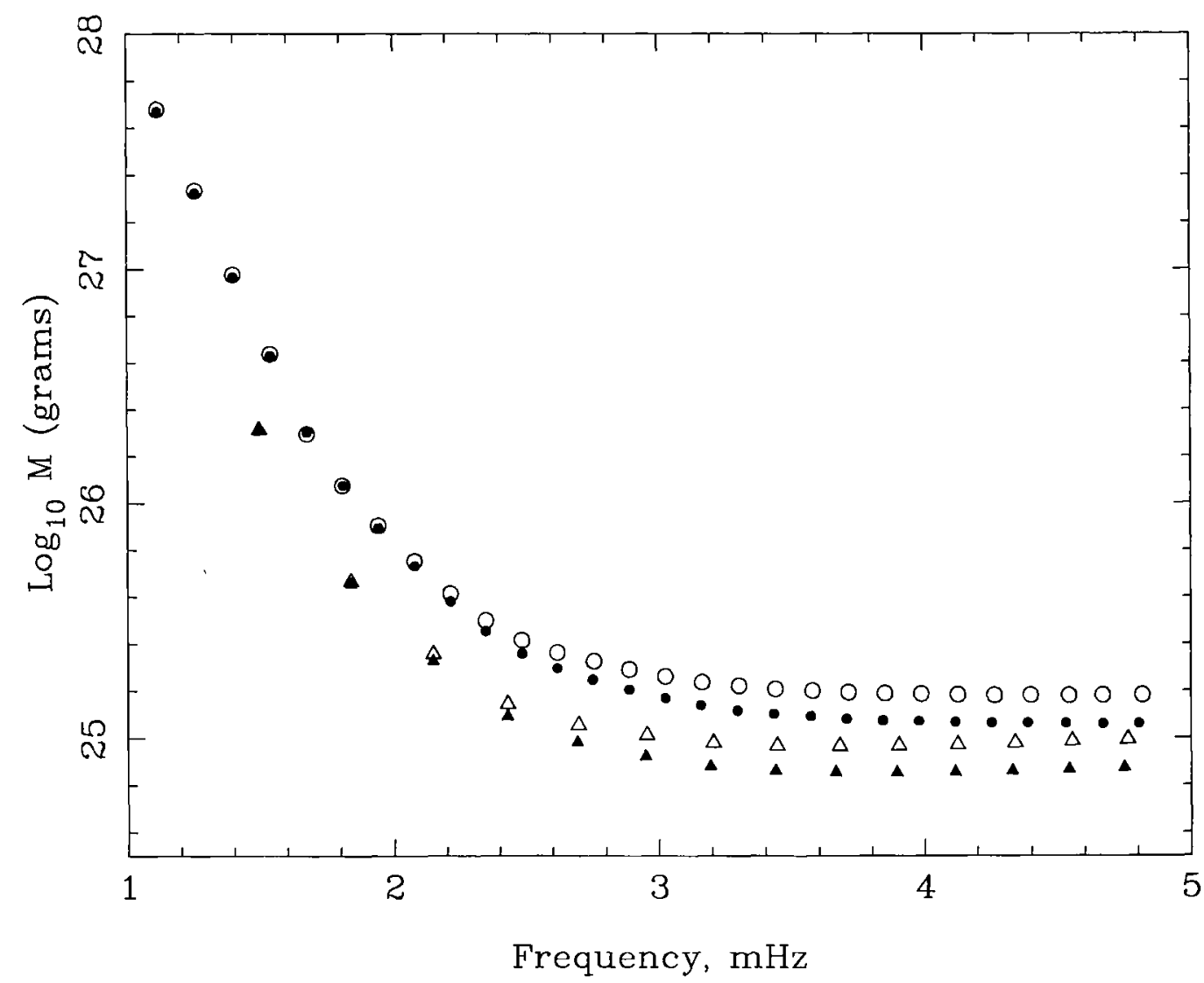

Fig. 16. Mode mass $M_{n l m}$, defined as the energy of a given mode divided by its mean-squared velocity, as calculated by P. Kumar for a standard solar model. A variety of different $n$ are shown for $l=0$ (circles) and $l=100$ (triangles); the open characters use the surface amplitude at $\tau_{5000}=0.5$, while the closed characters are for $\tau_{5000}=0.1$. For higher $l$, the mode mass at fixed frequency is roughly proportional to $l^{-1 / 2}$.

eigenfunctions have higher amplitudes in and above the photosphere, where the gas becomes optically thin and, therefore, very efficient at damping oscillatory motion. Here the assumption of a roughly constant energy input from convective turbulence, together with an energy drain $\dot{E} / E \sim \Gamma$, where $\Gamma$ is the measured mode linewidth, does an adequate job of reproducing the observed power spectra, as is shown in Figure 15.

It could also be pointed out that the mode energy does not depend strongly upon $l$, at least out to $l \simeq 200$ (Libbrecht et al., 1986). This is a result of the fact that only eddies in the uppermost layer of convection, the solar granulation, have turnover times fast enough to interact with the 5-min oscillations. Thus since the $\mathrm{p}$-mode eigenfunctions at a given frequency with different $l$ are nearly identical near the solar surface, it is expected and observed that their energies are also nearly identical. At very high $l$-values, preliminary observations indicate a falloff in the mode energies (Libbrecht et al., 1986). This may be an indication that 3-mode coupling is becoming an important source of damping, since it is expected that such coupling may kick in at high $l$ (Kumar and Goldreich, 1988).

Another observation which may be relevant to the mode excitation problem is that by Braun et al. (1987), showing that sunspots absorb incident acoustic energy at large wave numbers. The fact that the absorption is only seen at high $k_{h}$ can be understood from a simple argument by Kumar et al. (1988). Writing the high $k_{h}$ asymptotic 
dispersion relation as approximately

$$
\omega^{2}=\frac{3}{4} g k_{h}\left(n+\frac{3}{4}\right)
$$

we find that a wave packet has a group velocity

$$
v_{g}=\frac{\mathrm{d} \omega}{\mathrm{d} k_{h}}=\frac{\omega}{2 k_{h}} .
$$

Thus the time it takes the packet to traverse the sunspot is approximately

$$
t=\frac{k_{h} D}{\pi v}
$$

where $D$ is the sunspot diameter and $v$ is the mode frequency. (This assumes that the mode physics is the same inside and outside the spot, which is probably a somewhat poor assumption.) With this, any damping mechanism inside the spot will act for a longer time on high $k_{h}$ modes than low $k_{h}$, so the absorption is likely to be greater. Kumar et al. (1988) recently suggested that the magnetic field inside the sunspot could enhance the absorption of acoustic radiation by turbulence, while leaving the emission unaffected, thus accounting for the observations. If true, this is a remarkable confirmation of the stochastic turbulence p-mode excitation mechanism.

The stochastic excitation mechanism has yet to be applied to g-modes, but unless the energy per mode is enormously greater for g-modes than p-modes, the very large g-mode masses (see Christensen-Dalsgaard, 1986) suggest that the surface amplitudes would be very small indeed. A nucleo-thermal instability, based on the strong temperature sensitivity of ${ }^{3} \mathrm{He}$ burning in the solar core, has been proposed as a possible mechanism for exciting g-modes (see Press (1986) for a nice explanation of this process), but without firm observations of g-modes there is little else one can say.

Finally, it is worth pointing out in passing this example of how basic research, in this case the excitation of minute oscillations on the surface of the Sun, can bring about a better understanding of a process that is seen in a number of everyday phenomena. It is, after all, the excitation of acoustic waves by turbulence that we all know as airport noise.

\section{Extension to Other Stars}

Now that solar acoustic oscillations have been extensively studied, and appear to be the result of nothing more than turbulent convection in the solar envelope, we naturally ask whether similar oscillations can be observed in other stars. Christensen-Dalsgaard and Frandsen (1983) made a rough estimate of p-mode amplitudes expected on other stars, assuming excitation by turbulent convection, but without benefit of the more complete theory of Goldreich and Kumar. It was found that F-type stars could have oscillation amplitudes up to 10 times the solar amplitudes, primarily because of their extended atmospheres. Indeed a detection of p-modes on Procyon (spectral type F5IV) has been 
reported by Gelly et al. (1986), who find a mean velocity amplitude per mode of $70 \mathrm{~cm} \mathrm{~s}^{-1}$ in the range $1.17 \mathrm{mHz}<v<1.65 \mathrm{mHz}$, with a spacing between modes with $\Delta n=1$ of $79.4 \mu \mathrm{Hz}$. The mode spacing is in reasonable agreement with that expected from Procyon model calculations.

Unfortunately this detection, based on four nights of data using an atomic resonance filter on the $\mathrm{Na} \mathrm{D}$ lines, has not yet been confirmed by another run. However, if the detection is correct it is likely to be confirmed soon, as a fair amount of progress has been made in recent years toward developing the ideal instrument for measuring minute Doppler shifts. With a reasonably efficient echelle spectrograph, measuring Doppler shifts of all the spectral lines in a few thousand Angstroms of spectrum, it is likely that p-mode oscillations such as those reported for Procyon could be detected in stars as faint as sixth magnitude, perhaps fainter. The field of $\mathrm{p}$-mode seismology in stars other than the Sun is very much in its infancy, but it will be surprising if detections are not made on a number of bright stars soon. With such detections we will not only be able to probe the interior structure of our neighboring stars (for a discussion see Gough, 1985), but in addition we stand to perhaps learn a great deal about convection in the outer layers of these stars.

\section{The Future}

Although it is impossible to predict the future, one is tempted to extrapolate current trends to imagine where the fields of solar and stellar seismology are heading. The Global Oscillation Network Group (GONG) project is well underway, and we expect in the early 1990s to see a network of six Doppler imaging telescopes in operation around the world for the study of helioseismology. A number of space projects have also been proposed, which should provide excellent observations of the very low $l$ and high $l$ solar modes.

With these as well as other observations, there is no barrier to making measurements of solar p-mode frequencies which are an order of magnitude better than the best values currently available, and so one should expect to see such results in upcoming years. This will present an enormous challenge to solar model theorists, since the best models now match the observed frequencies to no better than a percent or so. A better solar model will undoubtedly result, but what is learned along the way about the solar helium abundance, opacities, equation of state, atmosphere structure, etc., cannot be foreseen.

Improved splitting measurements and inversion techniques will likely result in an inferred $v_{\odot}(r, \theta)$ which is accurate to a few percent throughout the convection zone. This will be an invaluable input for solar dynamo models, and one hopes that better progress will be made toward understanding the enigmatic solar dynamo. With these improved measurements we should also be able to make a better estimate of the solar core rotation rate.

The turbulent convection model for exciting the p-modes looks promising, but it is by no means firmly established as the only important excitation mechanism. Additional theoretical investigations into radiative transfer as a means of both exciting and damping 
the p-modes are greatly needed, as is a better understanding of the part played by 3-mode coupling to transfer energy between $\mathrm{p}$-modes. This entire area of $\mathrm{p}$-mode physics is a new and exciting part of helioseismology, and we expect to see much progress being made in the near future. The potential gain from this study is a better understanding of some aspects of convection in the Sun, using the interaction with p-modes as a probe.

A confirmed observation of g-modes in the Sun would be a boon to our study of the solar interior, since the g-mode frequencies are quite sensitive to the details of the solar interior structure in the core, unlike the p-modes. Theoretical predictions of g-modes amplitudes are not reliable, but it is not surprising that the amplitudes are smaller than current observations can unambiguously detect, owing to the fact that the g-modes are trapped far below the observed solar surface. Since the surface amplitudes of the modes are primarily horizontal and the g-mode frequencies are low, there is considerable solar noise from supergranulation, as was estimated by Duvall and Harvey (1986). This solar noise estimate is uncomfortably close to the current observational limits, so the prospects for observing g-modes are not rosy. Nevertheless, the benefits of g-mode measurements are great, so better observations and theoretical amplitude estimates are worth pursuing.

The localized interaction of p-modes with sunspots is being investigated, and one of the first firm results is the enhanced absorption mentioned above. One might go further and hope that p-modes could be used to probe the interior and depth structure of sunspots. This is an attractive possibility, and may produce interesting results in the future.

Oscillations in the brightness of the solar surface have not been studied in great detail yet, and the ease of the observations should encourage more work in this area shortly. These observations will not be easy to interpret, but they should provide additional information about the solar atmosphere, and may serve as an excellent check on the accuracy of our current solar atmosphere models.

Seismology of the Sun and stars is only just beginning and already a great deal has been learned about the fascinating phenomena of $\mathrm{p}$-mode oscillations. What in the $1960 \mathrm{~s}$ was thought to be incoherent local undulations of the solar surface is now seen as millions of coherent solar normal modes, all serving to probe the unseen depths of the solar interior.

\section{Acknowledgements}

I wish to express my gratitude for the hospitality and quiet office provided by the High Altitude Observatory, where most of this review was written. This work supported in part by NSF contract ATM-8604632.

\section{References}

Ando, H. and Osaki, Y.: 1975, Publ. Astron. Soc. Japan 27, 518.

Antia, H. M., Chitre, S. M., and Narashima, D.: 1986, Astrophys. Space Sci. 118, 169.

Bogdan, T. J. and Zweibel, E. G.: 1985, Astrophys. J. 298, 867. 
Braun, D. C., Duvall, T. L. Jr., and LaBonte, B. J.: 1987, Astrophys. J. 319, L27.

Brookes, J. R., Isaak, G. R., and van der Raay, H. B.: 1978, Monthly Notices Roy. Astron. Soc. 185, 1.

Brown, T. M.: 1985, in R. Ulrich (ed.), Proceedings of Snowmass Conference on Solar Seismology from Space, Jet Propulsion Laboratory, Pasadena.

Brown, T. M. and Morrow, C. A.: 1987, Astrophys. J. 314, L21.

Brown, T. M., Mihalas, B. W., and Rhodes, E. J., Jr.: 1986, in P. A. Sturrock (ed.), Physics of the Sun, Vol. I.,

D. Reidel Publ. Co., Dordrecht, Holland, p. 177.

Cacciani, A. and Fofi, M.: 1978, Solar Phys. 59, 179.

Christensen-Dalsgaard, J.: 1982, Monthly Notices Roy. Astron. Soc. 199, 735.

Christensen-Dalsgaard, J.: 1986, in D. O. Gough (ed.), Seismology of the Sun and Distant Stars, D. Reidel

Publ. Co., Dordrecht, Holland, p. 23.

Christensen-Dalsgaard, J. and Frandsen, S.: 1983, Solar Phys. 82, 469.

Christensen-Dalsgaard, J., Duvall, T. L., Gough, D. O., Harvey, J. W., and Rhodes, E. J., Jr.: 1985, Nature $315,378$.

Christensen-Dalsgaard, J., Gough, D., and Toomre, J.: 1985, Science 229, 923.

Claverie, A., Isaak, G. R., McLeod, C. P., van der Raay, H. B., and Roca Cortez, T.: 1979, Nature 282, 591.

Claverie, A., Isaak, G. R., McLeod, C. P., van der Raay, H. B., and Roca Cortez, T.: 1981a, Solar Phys. 74, 51.

Claverie, A., Isaak, G. R., McLeod, C. P., van der Raay, H. B., and Roca Cortez, T.: 1981b, Nature 293, 443.

Däppen, W., Gilliland, R. L., and Christensen-Dalsgaard, J.: 1986, Nature 321, 229.

Delache, P. and Scherrer, P. H.: 1983, Nature 306, 651.

Deubner, F.-L.: 1975, Astron. Astrophys. 44, 371.

Deubner, F.-L. and Gough, D.: 1984, Ann. Rev. Astron. Astrophys. 22, 593.

Dicke, R. H.: 1970, Ann. Rev. Astron. Astrophys. 8, 297.

Duvall, T. L., Jr.: 1982, Nature 300, 242.

Duvall, T. L., Jr. and Harvey J. W.: 1986, in D. O. Gough (ed.), Seismology of the Sun and Distant Stars, D.

Reidel Publ. Co., Dordrecht, Holland, p. 105.

Duvall, T. L., Harvey, J. W., Libbrecht, K. G., Popp, B. D., and Pomerantz, M. A.: 1988 Astrophys. J. (submitted).

Duvall, T. L., Jr., Harvey, J. W., and Pomerantz, M. A.: 1986, Nature 321, 500.

Evans, J. W. and Michard, R.: 1962, Astrophys. J. 136, 493.

Faulkner, J., Gough, D. O., and Vahia, M. N.: 1986, Nature 321, 226.

Gelly, B., Grec, G., and Fossat, E.: 1986, Astron. Astrophys. 164, 383.

Goldreich, P. and Keeley, D. K.: 1977a, Astrophys. J. 211, 934.

Goldreich, P. and Keeley, D. K.: 1977b, Astrophys. J. 212243.

Goldreich, P. and Kumar, P.: 1988, Astrophys. J. (submitted).

Goldreich, P., Kumar, P., and Libbrecht, K.: 1988, Astrophys. J. (submitted).

Gough, D. O.: 1981, Monthly Notices Roy. Astron. Soc. 196, 731.

Gough, D. O.: 1985, Nature 314, 14.

Gough, D. O. and Taylor, P. P.: 1984, in L. Belvedere and L. Paterno, (eds.), Oscillations as a Probe of the Sun's Interior, Memoire della Societa Astronomica Italiana Vol. 55, p. 69.

Grec, G., Fossat, E., and Pomerantz, M.: 1980, Nature 288, 541.

Grec, G., Fossat, E., and Pomerantz, M. A.: 1983, Solar Phys. 82, 55.

Howard, R.: 1984, Ann. Rev. Astron. Astrophys. 22, 131.

Isaak, G. R.: 1986, in D. O. Gough (ed.), Seismology of the Sun and Distant Stars, D. Reidel Publ., Dordrecht, Holland, p. 223.

Kidman, R. B. and Cox, A. N.: 1984, in R. K. Ulrich (ed.), Solar Seismology from Space, p. 317.

Kumar, P. and Goldreich, P.: 1988, Apstrophys. J. (submitted).

Kumar, P., Franklin, J., and Goldreich, P.: 1988, Astrophys. J. (submitted).

Lamb, H.: 1932, Hydrodynamics, 6th ed., Univ. Cambridge Press, Cambridge.

Leibacher, J. W., Noyes, R. W., Toomre, J., and Ulrich, R. K.: 1985, Sci. Am.

Leibacher, J. W. and Stein, R. F.: 1971, Astrophys. Letters 7, 191.

Leighton, R. B.: 1962, Astrophys. J. 135, 474.

Libbrecht, K. G.: 1988, in preparation. 
Libbrecht, K. G. and Kaufman, J. M.: 1988, Astrophys. J. (submitted).

Libbrecht, K. G. and Zirin, H.: 1986, Astrophys. J. 308, 413.

Libbrecht, K. G., Popp., B. D., Kaufman, J. M., and Penn, M. J.: 1986, Nature 323, 235.

Lightill, M. J.: 1952, Proc. Roy. Soc. A211, 564.

Lighthill, M. J.: 1954, Proc. Roy. Soc. A222, 1.

Lubow, S. H., Rhodes, E. J., Jr., and Ulrich, R. K.: 1980, in H. A. Hill and W. A. Dziembowski (eds.), Nonradial and Nonlinear Stellar Pulsation.

Nishikawa, J., Hamana, S., Mizugaki, K., and Hirayama, T.: 1986, Publ. Astron. Soc. Japan 38, 277.

Noels, A., Scuflaire, R., and Gabriel, M.: 1984, Astron. Astrophys. 130, 389.

Press, W. H.: 1986, in P. A. Sturrock (ed.), Physics of the Sun, Vol. I, D. Reidel Publ. Co., Dordrecht, Holland, p. 77.

Rust, D. M., Appourchaux, T., and Hill, F.: 1986, 'Advances in Helio- and Asteroseismology', in J. Christensen-Dalsgaard, (ed.), IAU Symp. 123.

Shibahashi, H., Noels, A., and Gabriel, M.: 1983, Astron. Astrophys. 123, 283.

Tassoul, M.: 1980, Astrophys. J. Suppl. 43, 469.

Toomre, J.: 1986, in D. O. Gough (ed.), Seismology of the Sun and Distant Stars, D. Reidel Publ. Co., Dordrecht, Holland, p. 1.

Ulrich, R. K.: 1970, Astrophys. J. 162, 993.

Ulrich, R. K. and Rhodes, E. J., Jr.: 1983, Astrophys. J. 265, 551.

Van der Raay, H. B.: 1984, in Theoretical Problems in Stellar Stability and Oscillations, University of Liège, p. 215.

Woodard, M. and Hudson, H. S.: 1983, Nature 305, 589.

Woodard, M. and Hudson, H. S.: 1984, Nature 309, 530.

Woodard, M. and Noyes, R. W.: 1985, Nature 318, 449. 